\title{
An Excess of Jupiter Analogs in Super-Earth Systems
}

\author{
Marta L. Bryan ${ }^{1}\left(\mathbb{0}\right.$, Heather A. Knutson $^{2}$, Eve J. Lee ${ }^{3}$,, B. J. Fulton ${ }^{1,4}{ }^{(10}$, Konstantin Batygin $^{2}$, Henry Ngo $^{5}$ (1), and \\ Tiffany Meshkat ${ }^{1}$ (D) \\ ${ }^{1}$ Cahill Center for Astronomy and Astrophysics, California Institute of Technology, 1200 East California Boulevard, MC 249-17, Pasadena, CA 91125, USA \\ ${ }^{2}$ Division of Geological and Planetary Sciences, California Institute of Technology, Pasadena, CA 91125, USA \\ ${ }^{3}$ TAPIR, Walter Burke Institute for Theoretical Physics, MC 350-17, California Institute of Technology, Pasadena, CA 91125, USA \\ ${ }^{4}$ IPAC-NASA Exoplanet Science Institute, Pasadena, CA 91125, USA \\ ${ }^{5}$ National Research Council of Canada, Herzberg Astronomy and Astrophysics, 5071 West Saanich Road, Victoria, BC, V9E 2E7, Canada \\ Received 2018 June 21; revised 2018 November 20; accepted 2018 November 27; published 2019 January 18
}

\begin{abstract}
We use radial velocity (RV) observations to search for long-period gas giant companions in systems hosting inner super-Earth $\left(1-4 R_{\oplus}, 1-10 M_{\oplus}\right)$ planets to constrain formation and migration scenarios for this population. We consistently refit published RV data sets for 65 stars and find nine systems with statistically significant trends indicating the presence of an outer companion. We combine these RV data with $\mathrm{AO}$ images to constrain the masses and semi-major axes of these companions. We quantify our sensitivity to the presence of long-period companions by fitting the sample with a power-law distribution and find an occurrence rate of $39 \% \pm 7 \%$ for companions $0.5-20 M_{\text {Jup }}$ and 1-20 au. Half of our systems were discovered by the transit method, and half were discovered by the RV method. While differences in the RV baselines and number of data points between the two samples lead to different sensitivities to distant companions, we find that occurrence rates of gas giant companions in each sample are consistent at the $0.5 \sigma$ level. We compare the frequency of Jupiter analogs in these systems to the equivalent rate from field star surveys and find that Jupiter analogs are more common around stars hosting super-Earths. We conclude that the presence of outer gas giants does not suppress the formation of inner super-Earths, and that these two populations of planets instead appear to be correlated. We also find that the stellar metallicities of systems with gas giant companions are higher than those without companions, in agreement with the well-established metallicity correlation from RV surveys of field stars.
\end{abstract}

Key words: methods: statistical - planetary systems - techniques: radial velocities

Supporting material: machine-readable table

\section{Introduction}

The presence or absence of outer gas giant planets can significantly influence the formation and evolution of planets on interior orbits. In our own solar system, Jupiter is thought to have played a key role in dynamically reshaping the outer solar system architecture after the dissipation of the gas disk (Tsiganis et al. 2005), driving volatile-rich planetesimals from beyond the ice line onto shorter-period orbits (O'Brien et al. 2006; Raymond 2006; Morbidelli et al. 2012; Raymond \& Izidoro 2017). At earlier times, the gap in the gas disk created by Jupiter's presence would also have suppressed the flow of solid materials into the inner disk where the terrestrial planets subsequently formed, affecting both the surface density of solids in the inner disk and the compositions of those solids (Morbidelli \& Crida 2007; Morbidelli et al. 2012, 2016; Lambrechts et al. 2014; Desch et al. 2018). It has even been theorized that an in-and-then-out-again migration by Jupiter and Saturn (Walsh et al. 2011) might have disrupted planet formation in the inner several au, therefore explaining why the solar system only hosts relatively small planets between 0.3 and 2 au and none interior to that (Batygin \& Laughlin 2015).

Given the dominant role that gas giant planets played in the early history of the solar system, it is natural to consider their possible influence in exoplanetary systems. Broadly speaking, there are several mechanisms by which outer gas giant planets can influence the formation and evolution of interior planets. Giant planets comparable to or larger than Saturn will open a gap in the gas disk (Lin \& Papaloizou 1986; Crida et al. 2006; Kley \& Nelson 2012), potentially suppressing the flow of small solids ("pebbles") to the inner disk. Moriarty \& Fischer (2015) found that the rate of planetesimal growth in the inner disk is sensitive to the rate at which pebbles drift radially inward, implying that systems with giant planets should have fewer and less massive planets in the inner region of the disk. However, the presence of a giant planet will also create local pressure maxima that collect solids, potentially sparking a secondary wave of planet formation (Whipple 1972; Masset et al. 2006; Rice et al. 2006; Hasegawa \& Pudritz 2011; Morbidelli \& Nesvorny 2012; Sato et al. 2016).

Through resonant transport associated with migration, gas giants can also dynamically excite the population of planetesimals from which rocky planets are forming, increasing the likelihood that collisions will result in disruption rather than accretion (Walsh et al. 2011; Batygin \& Laughlin 2015). However, unless this disrupted material is subsequently accreted onto the host star, this dynamical excitation and disruption of material is not a barrier to rocky planet formation (Wallace et al. 2017). Dynamically hot outer gas giants can perturb inner planets onto eccentric and/or inclined orbits, reducing the multiplicity of planets in those systems (Hansen 2017a; Pu \& Lai 2018) or leading to orbital instability within a few Myr in some extreme cases (Huang et al. 2017). These same gas giants can also act as a barrier that prevents smaller planets formed in the outer disk (i.e., beyond the orbit of the gas giants) from migrating inward (Izidoro et al. 2015).

Even if they do not directly influence the formation or dynamical evolution of inner planetary systems, the presence of an outer gas giant planet is in and of itself a statement about the 
properties of the primordial disk. In the core accretion model (Pollack et al. 1996), cores must form before the disk gas dissipates in order to acquire massive envelopes. The wellestablished correlation between gas giant planet frequency and stellar metallicity for Sun-like stars (Fischer \& Valenti 2005; Johnson et al. 2010) indicates that the core formation process occurs more readily in metal-rich disks (e.g., Dawson et al. 2015). The longer lifetime of disks around metal-rich stars also facilitates the formation of both gas giant planets (e.g., Ercolano \& Clarke 2010; Yasui et al. 2010) and those at lower masses (e.g., Buchhave et al. 2014; Petigura et al. 2018).

Despite the relative richness of theoretical work in this area, we currently have very few observational constraints on the role that outer gas giant planets play in determining the properties of inner planetary systems. This is largely due to the limited baselines of current surveys: both transit and radial velocity $(\mathrm{RV})$ surveys typically require the observation of one or more complete orbits in order to count a given signal as a secure detection, but even the longest-running surveys have baselines that are shorter than the orbital periods of the solar system gas giants (Cumming et al. 2008; Howard et al. 2010; Mayor et al. 2011; Dressing \& Charbonneau 2015; Bryan et al. 2016; Rowan et al. 2016; Wittenmyer et al. 2016). Recently, several RV surveys (Rowan et al. 2016; Wittenmyer et al. 2016) estimated the frequency of Jupiter analogs, which they defined as $0.3-13 M_{\text {Jup }}$ and 3-7 au (Wittenmyer et al. 2016) and $0.3-3 M_{\text {Jup }}$ and 3-6 au (Rowan et al. 2016), taking into account survey incompleteness at larger separations and smaller masses. Both surveys found the frequency of Jupiter analogs to be small; Wittenmyer et al. (2016) found an occurrence rate of $6.2 \%{ }_{-1.6 \%}^{+2.8 \%}$, while Rowan et al. (2016) found an occurrence rate of $\sim 3 \%$. However, neither of these surveys extended as far as Saturn's orbit, and relatively few of the stars in these two samples have known inner planets. Of the superEarth systems examined in this study, we find that only three were included in the Wittenmyer et al. (2016) sample, while Rowan et al. (2016) did not provide an explicit list of the stars included in their survey.

If we are willing to consider planet candidates with partially observed orbits, we can extend the statistical reach of these surveys to larger orbital separations. This also allows us to consider systems with inner transiting planets, which typically have shorter photometric and RV baselines (on the order of 1-5 yr; e.g., Marcy et al. 2014; Weiss \& Marcy 2014; Dressing et al. 2015; Astudillo-Defru et al. 2017). While the Kepler mission is, in principle, sensitive to transiting gas giant planets in Jupiter-like orbits (Foreman-Mackey et al. 2016; Uehara et al. 2016), the transit probability for these planets is extremely low, and a majority of the long-period planet candidates reported to date do not have inner transiting companions. Alternatively, long-term RV monitoring of systems with known inner planets can provide information on the frequency of outer companions regardless of whether or not they transit their host stars (e.g., Knutson et al. 2014; Montet et al. 2014; Bryan et al. 2016). Although our knowledge of the masses and orbital periods of these objects is incomplete, we can nonetheless search for correlations between inner planet properties and the presence or absence of an outer companion.

In previous studies, we considered the frequency of outer companions in systems with transiting hot Jupiters (Knutson et al. 2014) and with inner gas giant planets spanning a range of orbital periods (Bryan et al. 2016). In this study, we focus on stars known to host one or more super-Earth planets (defined as $1-4 R_{\oplus}$ or $1-10 M_{\oplus}$, depending on the detection method) located inside $0.5 \mathrm{au}$. These planets dominate the observed population of planets orbiting nearby stars, with $30 \%-50 \%$ of Sun-like stars hosting one or more super-Earths with orbital periods less than 100 days (Howard et al. 2010; Fressin et al. 2013; Petigura et al. 2013; Zhu et al. 2018a). We identify published RV data for a sample of 65 systems hosting inner super-Earths and use these data to search for long-period gas giant companions. In Section 2, we describe our sample of systems. In Section 3, we describe our fits to the RV data, identification of nonplanetary sources of RV trends, calculation of companion probability distributions, and completeness estimations. Finally, in Section 4, we discuss the occurrence rate of gas giant companions in our sample and implications of our results.

\section{Observations}

We collected published RV data for systems with at least one confirmed super-Earth, where we define a super-Earth as a planet with either a mass between 1 and $10 M_{\oplus}$ or a radius between 1 and $4 R_{\oplus}$, depending on the detection technique (Table 1). We exclude systems with fewer than 10 data points and baselines shorter than 100 days, leaving us with 65 systems that meet these criteria (Figure 1). Of that sample, 34 systems host at least one super-Earth discovered using the transit method, and 31 systems host at least one super-Earth discovered using the RV method. Eighteen of these systems are single-planet systems, while the remaining 47 are multiplanet systems. Forty-five planets have both measured masses and radii and thus measured densities. We provide a summary of the RV data used in this work in Table 1. We also include best-fit values for the RV acceleration from our orbital solution fitting as described in the following section in Table 1 . We list the complete set of individual RV measurements used in our analysis in Table 2 .

Our choice of $1-4 R_{\oplus}$ and $1-10 M_{\oplus}$ for inner "super-Earths" is physically motivated and results in a population that is unlikely to be contaminated with Neptune-mass planets. The shape of the planet occurrence rate as a function of orbital period changes drastically for planets smaller versus larger than $4 R_{\oplus}$. Whereas the number of smaller planets rises steeply out to $\sim 10$ days and plateaus beyond, larger planets grow more numerous with orbital periods out to at least $\sim 300$ days (Dong \& Zhu 2013; Petigura et al. 2018). This suggests that these two populations have distinct formation histories and motivates us to choose $4 R_{\oplus}$ as the upper bound on our sample of superEarths. ${ }^{6}$ For the subset of transiting planets with measured masses, those with radii larger than $4 R_{\oplus}$ are also typically much more massive than $10 M_{\oplus}$ (Petigura et al. 2017b). In order for planets to attain radii greater than $4 R_{\oplus}$, they must have gas-to-core mass ratios greater than $\sim 10 \%$ (Lopez \& Fortney 2014). In a gas-poor but not gas-empty environment that allows core assembly by giant impact (e.g., Lee \& Chiang 2016), only cores with masses $>\sim 10 M_{\oplus}$ are expected to end up with gas-to-core mass ratios $>\sim 10 \%$ (e.g., Lee \& Chiang 2015).

\footnotetext{
6 Although Fulton et al. (2017) recently reported evidence for a bimodal distribution in planet radius with peaks located at $1-1.7$ and $1.7-4 R_{\oplus}$, current evidence suggests that this bimodality is not an outcome of divergent formation histories but is instead likely driven by photoevaporative mass loss on a subset of the most highly irradiated planets (Owen \& Wu 2013, 2017).
} 
Table 1

Sample of Systems

\begin{tabular}{|c|c|c|c|c|c|c|c|c|}
\hline Target $^{\mathrm{a}}$ & $M_{\star}\left(M_{\odot}\right)$ & {$[\mathrm{Fe} / \mathrm{H}]^{\mathrm{b}}$} & $N_{\mathrm{pl}}$ & Disc. Method & $N_{\text {obs }}$ & Baseline (days) & Trend $\left(\mathrm{m} \mathrm{s}^{-1} \mathrm{yr}^{-1}\right)$ & References \\
\hline Corot-7 & $0.93 \pm 0.03$ & $0.09 \pm 0.01$ & 2 & Transit & 109 & 357 & $10.95 \pm 7.30$ & $(9),(58)$ \\
\hline Corot-24 & $0.91 \pm 0.09$ & $0.30 \pm 0.15$ & 2 & Transit & 50 & 1154 & $-10.95 \pm 2.92$ & (10) \\
\hline HD 3167 & $0.86 \pm 0.03$ & $0.04 \pm 0.05$ & 3 & Transit & 251 & 152 & $\mathbf{9 . 9 6}_{-2.04}^{+1.97}$ & (37) \\
\hline $\mathrm{K} 2-3$ & $0.61 \pm 0.09$ & $-0.32 \pm 0.13$ & 3 & Transit & 72 & 103 & $10.95_{-7.67}^{+6.94}$ & (12) \\
\hline $\mathrm{K} 2-32$ & $0.86 \pm 0.03$ & $-0.02 \pm 0.01$ & 3 & Transit & 74 & 441 & $2.41_{-2.01}^{+2.04}$ & (14), (59) \\
\hline Kepler-10 & $0.91 \pm 0.02$ & $-0.11 \pm 0.04$ & 2 & Transit & 148 & 510 & $3.72_{-1.97}^{+2.04}$ & $(8),(48)$ \\
\hline Kepler-20 & $0.91 \pm 0.03$ & $0.11 \pm 0.04$ & 6 & Transit & 134 & 2262 & $-2.01 \pm-1.06$ & $(13),(48),(57)$ \\
\hline Kepler-21 & $1.41_{-0.03}^{+0.02}$ & $-0.04 \pm 0.04$ & 1 & Transit & 122 & 1756 & $0.73 \pm 1.05$ & $(3),(4),(48)$ \\
\hline Kepler-22 & $0.97 \pm 0.06$ & $-0.20 \pm 0.04$ & 1 & Transit & 16 & 373 & $0.84_{-3.32}^{+3.13}$ & $(5),(48)$ \\
\hline Kepler-25 & $1.19 \pm 0.06$ & $-0.05 \pm 0.04$ & 3 & Transit & 62 & 828 & $2.23_{-2.30}^{+2.41}$ & $(2),(48)$ \\
\hline Kepler-37 & $0.80 \pm 0.07$ & $-0.25 \pm 0.04$ & 3 & Transit & 33 & 862 & $0.26 \pm 1.06$ & (2), (48) \\
\hline Kepler-48 & $0.88 \pm 0.06$ & $0.26 \pm 0.04$ & 4 & Transit & 28 & 1135 & $2.01_{-3.32}^{+3.10}$ & $(2),(48)$ \\
\hline Kepler-62 & $0.69 \pm 0.02$ & $-0.34 \pm 0.04$ & 5 & Transit & 13 & 128 & $60.2_{-32.0}^{+42.0}$ & $(15),(48)$ \\
\hline Kepler-68 & $1.08 \pm 0.05$ & $0.14 \pm .04$ & 3 & Transit & 64 & 1207 & $1.68_{0.803}^{+0.77}$ & $(2),(48)$ \\
\hline Kepler-93 & $0.91 \pm 0.03$ & $-0.16 \pm 0.02$ & 1 & Transit & 118 & 1892 & $12.01 \pm 0.44$ & (1), (2), (58) \\
\hline Kepler-94 & $0.81 \pm 0.06$ & $0.32 \pm 0.04$ & 2 & Transit & 29 & 799 & $28.11_{-20.44}^{+18.62}$ & $(2),(48)$ \\
\hline Kepler-95 & $1.08 \pm 0.08$ & $0.24 \pm 0.02$ & 1 & Transit & 31 & 1078 & $0.62_{-1.13}^{+1.17}$ & (2), (58) \\
\hline Kepler-96 & $1.00 \pm 0.06$ & $0.07 \pm 0.02$ & 1 & Transit & 26 & 772 & $-1.50_{-1.10}^{+1.17}$ & (2), (58) \\
\hline Kepler-97 & $0.94 \pm 0.06$ & $-0.21 \pm 0.02$ & 1 & Transit & 20 & 789 & $-\mathbf{4 . 4 9}_{-1.35}^{+1.31}$ & (2), (58) \\
\hline Kepler-98 & $0.99 \pm 0.06$ & $0.13 \pm 0.02$ & 1 & Transit & 22 & 805 & $2.34_{-2.04}^{+2.15}$ & (2), (58) \\
\hline Kepler-99 & $0.79 \pm 0.06$ & $0.27 \pm 0.01$ & 1 & Transit & 21 & 792 & $-2.96_{-1.39}^{+1.35}$ & (2), (48) \\
\hline Kepler-100 & $1.08 \pm 0.06$ & $0.10 \pm 0.02$ & 3 & Transit & 49 & 1221 & $1.06 \pm 0.80$ & $(2),(58)$ \\
\hline Kepler-102 & $0.81 \pm 0.06$ & $0.13 \pm 0.01$ & 5 & Transit & 35 & 897 & $1.06_{-1.10}^{+1.13}$ & $(2),(58)$ \\
\hline Kepler-103 & $1.09 \pm 0.07$ & $0.13 \pm 0.02$ & 2 & Transit & 19 & 736 & $2.70 \pm 1.79$ & (2), (58) \\
\hline Kepler-106 & $1.00 \pm 0.06$ & $-0.07 \pm 0.02$ & 4 & Transit & 25 & 1074 & $-0.96 \pm 1.3$ & $(2),(58)$ \\
\hline Kepler-109 & $1.04 \pm 0.06$ & $-0.01 \pm 0.02$ & 2 & Transit & 15 & 1092 & $-2.59_{-2.81}^{+2.48}$ & $(2),(58)$ \\
\hline Kepler-113 & $0.75 \pm 0.06$ & $0.16 \pm 0.01$ & 2 & Transit & 24 & 833 & $0.15 \pm 3.65$ & $(2),(58)$ \\
\hline Kepler-131 & $1.02 \pm 0.06$ & $0.15 \pm 0.02$ & 2 & Transit & 20 & 742 & $0.073_{-2.19}^{+2.11}$ & $(2),(58)$ \\
\hline Kepler-406 & $1.07 \pm 0.06$ & $0.23 \pm 0.02$ & 2 & Transit & 42 & 801 & $0.73 \pm 1.10$ & $(2),(58)$ \\
\hline Kepler-407 & $1.00 \pm 0.06$ & $0.35 \pm 0.02$ & 1 & Transit & 17 & 750 & $-\mathbf{1 5 6 . 5 9} \pm 4.02$ & $(2),(58)$ \\
\hline Kepler-409 & $0.92 \pm 0.06$ & $0.05 \pm 0.01$ & 1 & Transit & 25 & 175 & $8.76 \pm 6.21$ & $(2),(58)$ \\
\hline Kepler-454 & $1.03_{-0.03}^{+0.04}$ & $0.22 \pm 0.02$ & 2 & Transit & 102 & 1901 & $\mathbf{1 4 . 5 6}_{-0.62}^{+0.58}$ & $(11),(58)$ \\
\hline LHS 1140 & $0.15 \pm 0.02$ & $-0.24 \pm 0.10$ & 1 & Transit & 144 & 386 & $0.44 \pm 1.68$ & (6) \\
\hline WASP-47 & $0.99 \pm 0.05$ & $0.36 \pm 0.05$ & 4 & Transit & 146 & 2340 & $-1.31_{-2.26}^{+1.28}$ & $(45),(46),(47)$ \\
\hline $55 \mathrm{Cnc}$ & $0.94 \pm 0.05$ & $0.36 \pm 0.01$ & 5 & RV & 1126 & 8476 & $-0.33_{-0.15}^{+0.16}$ & $(16),(17),(56),(59)$ \\
\hline $61 \mathrm{Vir}$ & $0.95_{-0.03}^{+0.04}$ & $-0.04 \pm 0.01$ & 3 & $\mathrm{RV}$ & 786 & 7060 & $-0.31 \pm 0.14$ & $(18),(19),(56),(59)$ \\
\hline GJ $15 \mathrm{~A}$ & $0.38 \pm 0.06$ & $-0.32 \pm 0.17$ & 1 & $\mathrm{RV}$ & 349 & 6215 & $-0.44_{-0.073}^{+0.077}$ & $(19),(20)$ \\
\hline GJ $163^{c}$ & $0.40 \pm 0.04$ & $-0.01 \pm 0.10$ & 3 & RV & 153 & 3068 & $-0.12 \pm 0.16$ & (41) \\
\hline GJ 176 & 0.50 & $0.15 \pm 0.17$ & 1 & $\mathrm{RV}$ & 167 & 5836 & $-0.27_{-0.35}^{+0.34}$ & $(19),(21),(53)$ \\
\hline GJ 273 & 0.29 & $-0.17 \pm 0.17$ & 2 & RV & 354 & 6855 & $\mathbf{1 . 2 _ { - 0 . 0 6 2 } ^ { + 0 . 0 6 6 }}$ & (19), (22), (53) \\
\hline GJ 433 & 0.48 & $-0.22 \pm 0.10$ & 1 & RV & 100 & 5476 & $-0.22_{-0.20}^{+0.22}$ & $(19),(23)$ \\
\hline GJ 536 & $0.52 \pm 0.05$ & $-0.08 \pm 0.09$ & 1 & RV & 228 & 6128 & $-0.13 \pm 0.10$ & $(19),(24)$ \\
\hline GJ $581^{\mathrm{c}}$ & $0.31 \pm 0.02$ & $-0.10 \pm 0.17$ & 3 & $\mathrm{RV}$ & 531 & 5139 & $0.43_{-0.15}^{+0.16}$ & $(19),(25),(53)$ \\
\hline GJ $667 \mathrm{C}^{\mathrm{c}}$ & $0.33 \pm 0.02$ & $-0.59 \pm 0.10$ & 5 & $\mathrm{RV}$ & 238 & 4847 & $1.79 \pm \mathbf{0 . 1 8}$ & $(19),(26),(27),(54)$ \\
\hline GJ 676 & $0.71 \pm 0.04$ & $0.23 \pm 0.10$ & 3 & RV & 127 & 3231 & $\mathbf{0} \pm \mathbf{0}^{\mathrm{d}}$ & (44) \\
\hline GJ 832 & $0.45 \pm 0.05$ & $-0.30 \pm 0.20$ & 2 & $\mathrm{RV}$ & 109 & 5569 & $0.14_{-0.22}^{+0.21}$ & $(32)$ \\
\hline GJ $876^{\mathrm{c}}$ & $0.33 \pm 0.03$ & $0.19 \pm 0.17$ & 4 & RV & 401 & 6762 & $1.04 \pm 0.38$ & (19), (39), (53), (56) \\
\hline GJ 3138 & 0.68 & $-0.30 \pm 0.12$ & 3 & RV & 199 & 2932 & $0.20_{-0.13}^{+0.12}$ & (22) \\
\hline GJ 3293 & 0.42 & $0.02 \pm 0.09$ & 4 & $\mathrm{RV}$ & 205 & 2311 & $-0.11 \pm 0.11$ & (22) \\
\hline GJ 3323 & 0.16 & $-0.06 \pm 0.17$ & 2 & RV & 142 & 4333 & $0.12 \pm 0.11$ & $(22),(53)$ \\
\hline GJ 3341 & 0.47 & $-0.09 \pm 0.09$ & 1 & $\mathrm{RV}$ & 135 & 1456 & $0.27 \pm 0.20$ & $(28)$ \\
\hline GJ 3634 & $0.45 \pm 0.05$ & $-0.10 \pm 0.10$ & 1 & RV & 54 & 460 & $9.6_{-1.0}^{+0.95}$ & (43) \\
\hline GJ 3998 & $0.50 \pm 0.05$ & $-0.16 \pm 0.09$ & 2 & $\mathrm{RV}$ & 136 & 869 & $-0.66_{-0.51}^{+0.55}$ & $(38)$ \\
\hline HD 1461 & 1.02 & $0.19 \pm 0.01$ & 2 & $\mathrm{RV}$ & 921 & 6310 & $-0.055_{-0.15}^{+0.16}$ & (19), (35) \\
\hline HD 7924 & $0.83_{-0.04}^{+0.02}$ & $-0.15 \pm 0.01$ & 3 & RV & 906 & 4783 & $0.080 \pm 0.051$ & (34), (59) \\
\hline HD 20794 & 0.70 & $-0.40 \pm 0.01$ & 4 & $\mathrm{RV}$ & 187 & 2610 & $-0.044_{-0.044}^{+0.047}$ & (31) \\
\hline $\mathrm{HD} 40307^{\mathrm{c}}$ & 0.77 & $-0.31 \pm 0.03$ & 5 & RV & 226 & 3811 & $0.55 \pm 0.040$ & (35) \\
\hline HD 85512 & 0.69 & $-0.33 \pm 0.03$ & 1 & $\mathrm{RV}$ & 185 & 2745 & $0.32 \pm 0.051$ & $(31)$ \\
\hline HD $156668^{c}$ & $0.77 \pm 0.02$ & $0.05 \pm 0.06$ & 1 & $\mathrm{RV}$ & 527 & 4226 & $-0.15 \pm 0.12$ & $(30),(52)$ \\
\hline HD $175607^{c}$ & $0.74 \pm 0.05$ & $-0.62 \pm 0.01$ & 1 & $\mathrm{RV}$ & 110 & 3390 & $0.13_{-0.12}^{+0.11}$ & (40) \\
\hline
\end{tabular}


Table 1

(Continued)

\begin{tabular}{lcccccccc}
\hline \hline Target $^{\mathrm{a}}$ & $M_{\star}\left(M_{\odot}\right)$ & {$[\mathrm{Fe} / \mathrm{H}]^{\mathrm{b}}$} & $N_{\mathrm{pl}}$ & Disc. Method & $N_{\text {obs }}$ & Baseline (days) & ${\text { Trend }\left(\mathrm{m} \mathrm{s}^{-1} \mathrm{yr}^{-1}\right)}^{\mathrm{References}}$ \\
\hline HD 181433 & 0.78 & $0.33 \pm 0.13$ & 3 & $\mathrm{RV}$ & 107 & 1757 & $1.5_{-2.9}^{+0.98}$ \\
HD 215497 & $0.87 \pm 0.02$ & $0.23 \pm 0.07$ & 2 & $\mathrm{RV}$ & 99 & 1842 & $-0.24 \pm 0.23$ & $(33)$ \\
HD 219134 & $0.81 \pm 0.02$ & $0.11 \pm 0.04$ & 6 & $\mathrm{RV}$ & 1033 & 7421 & $-0.45 \pm-0.08$ & $(19),(42),(56)$ \\
Proxima Cen & $0.12 \pm 0.02$ & $0.22 \pm 0.03$ & 1 & $\mathrm{RV}$ & 144 & 4325 & $-0.13_{-0.095}^{+0.10}$ & $(36),(51),(55)$ \\
Wolf 1061 & 0.29 & $-0.02 \pm 0.17$ & 3 & $\mathrm{RV}$ & 187 & 4136 & $0.037_{-0.055}^{+0.058}$ & $(22),(53)$ \\
\hline
\end{tabular}

Notes.

a Systems in bold have statistically significant long-term trends.

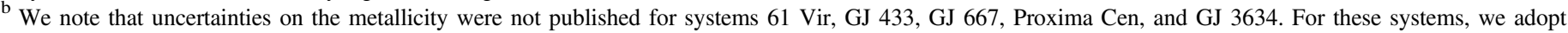
metallicity uncertainties of 0.1 dex.

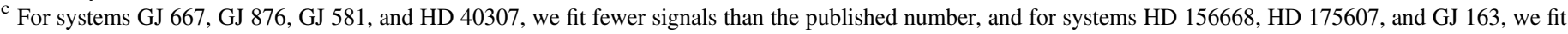
additional signals. See Section 3.1 for details.

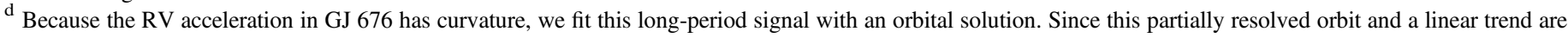
degenerate, we fix the linear trend term in this fit to zero, as well as the eccentricity of this outer companion.

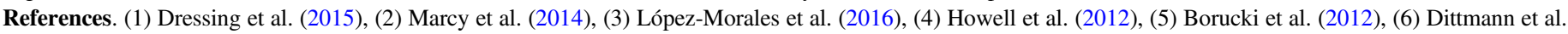

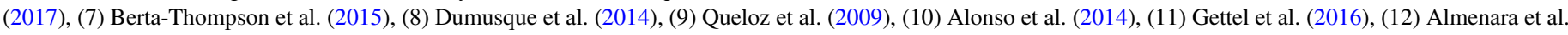

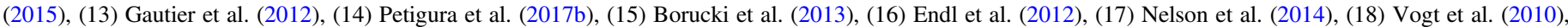

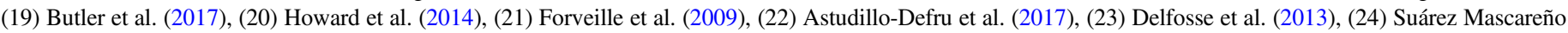

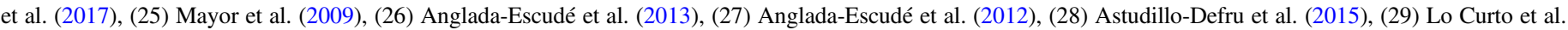

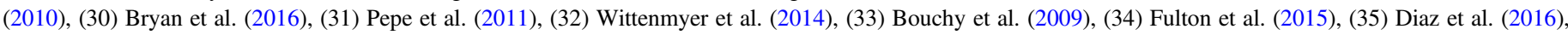

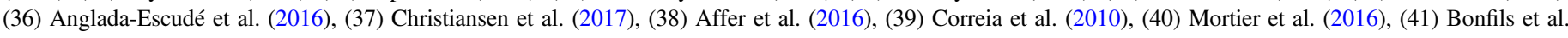

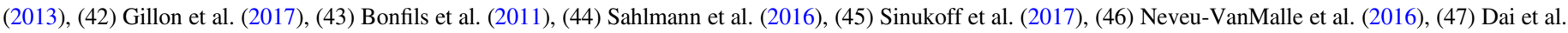

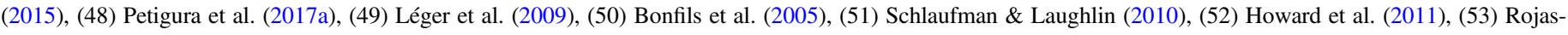

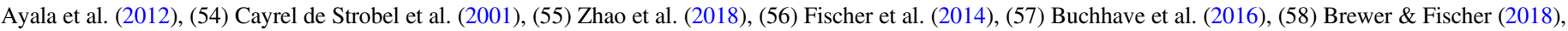
(59) Brewer et al. (2016).

We next check whether our sample is likely to be contaminated by planets whose true masses are comparable to or larger than that of Neptune $\left(17 M_{\oplus}\right)$. Looking at the transiting sample first, we find that 26 systems have at least one super-Earth with a mass measurement (note that these are true mass measurements as opposed to $m \sin i$ ). We find that all 26 of these systems have a super-Earth below Neptune mass $\left(17 M_{\oplus}\right)$. With regard to the RV mass cutoff of $m \sin i<$ $10 M_{\oplus}$, we quantify what percentage of these planets might have true masses comparable to or larger than that of Neptune. We drew $10^{6}$ sets of inclinations from a uniform distribution in $\cos (i)$, calculating true masses for each super-Earth in the RV sample. When there was more than one super-Earth in a system, we selected the lower-mass one. We found that $5 \%$ of these mass values were above $17 M_{\oplus}$, whereas $95 \%$ were below Neptune mass. We therefore conclude that it is unlikely that our sample contains any Neptune-mass planets.

\section{Analysis \\ 3.1. RV Fitting}

The presence of a distant companion manifests as a longterm trend in the RV data when the orbital period of the companion is significantly longer than the RV baseline. In order to quantify the significance of these long-term trends, we simultaneously fit for the orbits of the known inner planets, as well as a linear trend in each data set using RadVel (Fulton et al. 2018). After identifying the best-fit solution for each data set, we next carry out a Markov chain Monte Carlo (MCMC) exploration of the parameter space to determine the uncertainties on each model parameter. For a system with a single known planet, our model has eight free parameters, including six orbital parameters (the planet's velocity semiamplitude, orbital period, eccentricity, argument of periastron, true anomaly, and an RV zero-point), a linear velocity trend, and stellar jitter.

We fit using the basis $\left[P, T_{c}, \sqrt{e} \sin \omega, \sqrt{e} \cos \omega, K\right]$ and impose flat priors on all of these orbital elements. For the planets that transit, we apply Gaussian priors centered on the orbital period and time of conjunction values derived from the transit data with a width equal to the measured uncertainties on these values. In cases where we include data from multiple telescopes or the HIRES data include observations taken prior to the 2004 detector upgrade (Vogt et al. 2005; Bryan et al. 2016), we fit a separate RV zero-point and jitter value for each data set. We do not include data sets that have fewer than 10 data points. We also bin each set of RV data in $2 \mathrm{hr}$ increments, binning data sets from different telescopes separately. We define our likelihood function in Equation (1), where $\sigma_{i}$ is the instrumental error, $\sigma_{\mathrm{jit}}$ is the stellar jitter, $v$ is the data, and $m$ is the model:

$$
\mathscr{L}=\prod_{i} \frac{1}{\sqrt{2 \pi} \sqrt{\sigma_{i}^{2}+\sigma_{\mathrm{jit}}^{2}}} \exp \left(-0.5\left(\frac{\left(v_{i}-m_{i}\right)^{2}}{\sigma_{i}^{2}+\sigma_{\mathrm{jit}}^{2}}\right)\right) .
$$

We initialize each MCMC chain using the best-fit parameters from our fit. We note that for several systems, we fit a different number of Keplerian orbits than the published number of planets (Table 1). Of the transiting planet systems, this includes Kepler-20 and Kepler-407. For Kepler-20, there are six published planets, but we fit four Keplerian orbits, as two of the transiting planets did not yield statistically significant RV semi-amplitudes in previous studies (Buchhave et al. 2016). For Kepler-407, there is one transiting planet and a long-term trend with curvature that was published as a planet detection, but we only fit a full Keplerian orbit for the inner planet, as the outer planet's orbital period is poorly constrained by the current RV data (Marcy et al. 2014). 

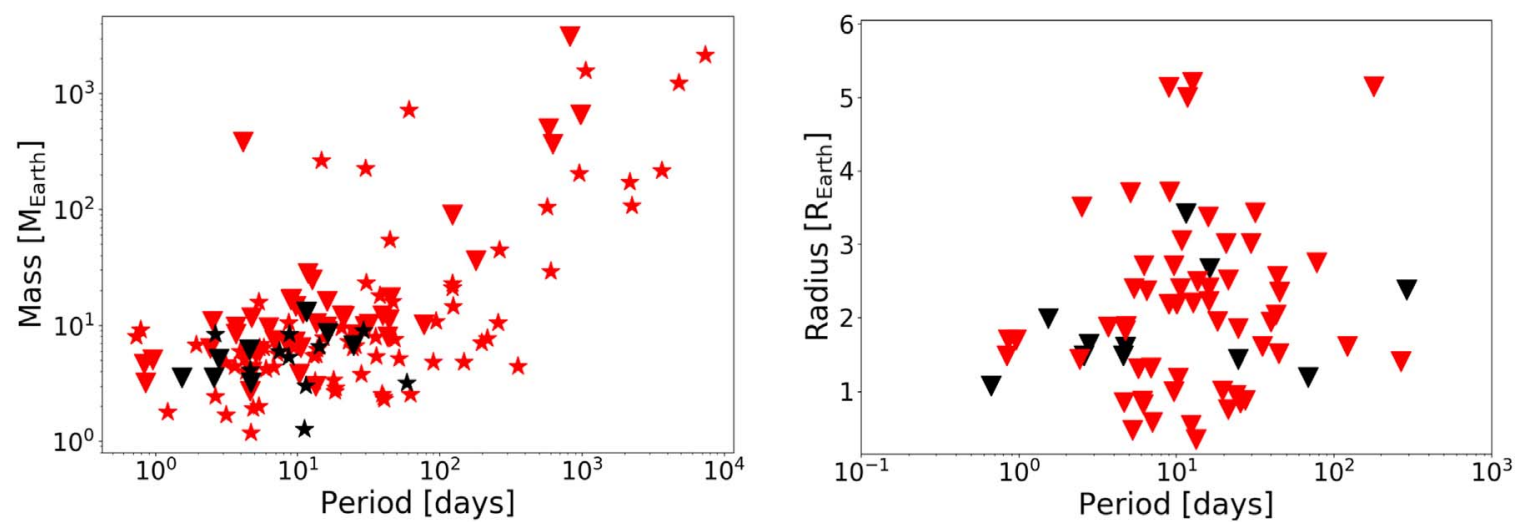

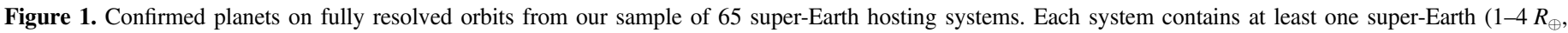

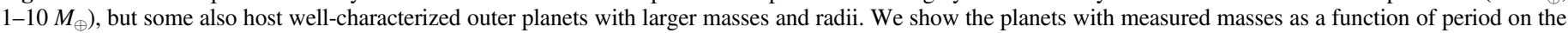

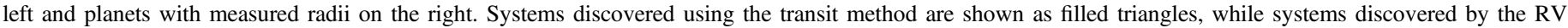
method are shown as filled stars. Multiplanet systems are plotted in red, while single planets are plotted in black.

Table 2

Published RVs Used in This Study

\begin{tabular}{cccc}
\hline \hline System & JD & $\mathrm{RV}\left(\mathrm{m} \mathrm{s}^{-1}\right)$ & $\sigma_{\mathrm{RV}}\left(\mathrm{m} \mathrm{s}^{-1}\right)$ \\
\hline Corot-7 & $2,454,527.5$ & $31,181.8$ & 1.5 \\
Corot-7 & $2,454,530.6$ & $31,173.2$ & 1.5 \\
Corot-7 & $2,454,550.5$ & $31,197.2$ & 2.1 \\
Corot-7 & $2,454,775.8$ & $31,188.1$ & 2.2 \\
Corot-7 & $2,454,776.7$ & $31,184.8$ & 2.8 \\
Corot-7 & $2,454,778.7$ & $31,181.7$ & 2.0 \\
Corot-7 & $2,454,779.7$ & $31,173.4$ & 1.6 \\
Corot-7 & $2,454,780.7$ & $31,175.0$ & 2.2 \\
Corot-7 & $2,454,789.8$ & $31,180.5$ & 2.6 \\
Corot-7 & $2,454,790.8$ & $31,187.8$ & 1.6 \\
\hline
\end{tabular}

Note. The full set of RVs for each of these systems are available as electronic tables online.

(This table is available in its entirety in machine-readable form.)

For the RV-detected planetary systems, we search for periodic signals in the RV data sets using the automated planet search pipeline described in Fulton et al. (2015). We fit Keplerian orbits to all signals with empirical false-alarm probabilities (eFAP) less than or equal to $1 \%$ and $K$ greater than or equal to $1.0 \mathrm{~m} \mathrm{~s}^{-1}$ in our final RV analysis. For systems GJ 667, GJ 876, GJ 581, and HD 40307, we find that we are only able to recover a subset of the previously published planets and fit for two, three, two, and four planetary orbits, respectively, in these systems.

For GJ 3341, HD 156668, HD 175607, and GJ 163, we find additional periodic signals with eFAP $\leqslant 1 \%$ that do not correspond to the periods of the confirmed planets in these systems and may be due to either stellar activity or additional unconfirmed planetary companions. We determine whether to include these additional periodic signals by comparing model fits with and without these additional signals using the Bayesian information criterion (BIC). The BIC is defined as $\mathrm{BIC}=-2 L+k \ln n$, where $L$ is the log likelihood of a model fit, $k$ is the number of free parameters in the model, and $n$ is the number of data points. In this case, the preferred model is the one with the lowest BIC value. If the BIC value for the model with additional periodic signals is smaller than the BIC value for the model without the additional periodic signals by at least 10 (a reasonable rule of thumb for statistically significant improvements in fit; Kass \& Raftery 1995), we consider the model with additional periodic signals to be a better fit and include these signals in subsequent analyses.

For GJ 3341, using the automated planet search pipeline, we recover the known 14.2 day period planet and also detect a second signal with a period of 202 days and an amplitude of $2.0 \mathrm{~m} \mathrm{~s}^{-1} \quad(\mathrm{eFAP}=1 \%)$. When we compare BIC values between model fits to the RV data with and without this 202 day signal, we find that the BIC value for the model with the additional periodic signal is only slightly smaller than the $\mathrm{BIC}$ value for the model without the additional periodic signal $(\triangle \mathrm{BIC}=3.9)$. We therefore do not include this additional periodic signal in the RV model fits to the data.

For HD 156668, the known planet with a period of 4.6 days is easily detected by our automated pipeline. We also detect a second signal at a period of 808 days with an amplitude of $2.9 \mathrm{~m} \mathrm{~s}^{-1}$ and a very low false-alarm probability. This appears to be a promising planet candidate but will require additional vetting in order to assess its planetary nature. When comparing model fits, we find that $\triangle \mathrm{BIC}=99.9$ between the model without the additional signal and the model with the additional signal; thus, we include this 808 day signal in our RV model fits. If real, this signal would correspond to a planet with $M \sin i=31 M_{\oplus}$.

For HD 175607, which has a known planet with an orbital period of 29 days, we detect a second signal with an eFAP of $0.5 \%$ and a period of 707 days. However, this period is very close to $2 \mathrm{yr}$ and has poor phase coverage as a result. We also see a third peak in the periodogram at double this period ( $\sim 1400$ days), indicating that there is some ambiguity in the true period of this signal. When comparing model fits, we find that $\triangle \mathrm{BIC}=27.4$ between the model without the additional signal and the model with the additional signal; thus, we include this 707 day signal in our RV model fits. We note, however, that this additional signal will likely require additional RV observations to confirm or disprove its planetary nature. If real, this signal would correspond to a planet with $M \sin i=24 M_{\oplus}$.

For GJ 163, we detect signals corresponding to the three previously confirmed planets, as well as two additional signals at periods of 19 and 108 days. Bonfils et al. (2013) previously identified these two signals as potential planet candidates. We find that a model including these additional signals is a significantly better fit to the RV data than a model that does not 
include these signals $(\triangle \mathrm{BIC}=12.1)$, so we include all five signals in our RV analysis.

When comparing orbital solutions for the RV sample, we found that four of these systems had orbital solutions that were inconsistent at the $>2 \sigma$ level with published values: 61 Vir, GJ 273, GJ 667, and GJ 876. For 61 Vir, the published orbital solution came from fits to $206 \mathrm{RVs}$ with no linear trend included (Vogt et al. 2010), whereas in our fits, we use 786 RVs and include a linear trend (significance $2.2 \sigma$ ). For GJ 273, the published orbital solution utilizes a data set of $279 \mathrm{RVs}$ and does not fit a linear trend. We include an additional 75 data points from Butler et al. (2017) in our fits and detect a linear trend with a significance of $19 \sigma$. For GJ 667, the published solution uses seven Keplerian orbits (five confirmed planets, two additional signals) and a linear trend to fit a total of $214 \mathrm{RV}$ measurements. In our blind planet search, we only recover three of the seven signals using a marginally bigger RV data set (238 measurements in total) and including a linear trend. Finally, GJ 876 is a dynamically rich system with three planets in a Laplace resonance. Previous studies fit the RV data for this star using an $\mathrm{N}$-body code with four planets, while we fit these data using Keplerian orbits for the three inner planets (our blind planet search did not identify the fourth planet).

After fitting our model to each data set, we search for systems with statistically significant linear trends (defined here as fits where the linear slope differs from zero by more than $3 \sigma)$. We list the best-fit trend values from the maximumlikelihood fit for each system in Table 1, with corresponding uncertainties determined from the MCMC chains. We find that 14 of the 65 systems in our sample have statistically significant trends. We used the BIC to determine whether these statistically significant long-term trends were best modeled with a linear trend, a quadratic trend, or an additional Keplerian orbit. In all cases but one (GJ 676), we found that a linear trend was the preferred model. For GJ 676, the curvature of the trend was significant enough to justify a fit with a full Keplerian orbital model.

We also find nine systems in our sample with fully resolved outer gas giant companions that were previously identified in the published literature. For the purposes of this study, we define an outer "gas giant" as a companion with a mass greater than $0.5 M_{\text {Jup }}$ outside of $1 \mathrm{au}$. Although some super-Earths in our sample also have outer companions with masses smaller than this cutoff, these planets were likely too small to open a gap in the protoplanetary gas disk (e.g., Lin \& Papaloizou 1986; Crida et al. 2006; Kley \& Nelson 2012). The 1 au cutoff includes all gas giants $>0.5 M_{\text {Jup }}$ orbiting exterior to lowermass planets and excludes four gas giant planets orbiting at smaller separations (55 Cnc b at $0.11 \mathrm{au}$; GJ $876 \mathrm{~b}$ and $\mathrm{c}$ at 0.21 and $0.13 \mathrm{au}$, respectively; and WASP-47 b at $0.05 \mathrm{au}$ ).

We list the properties of these previously confirmed outer gas giant planets in Table 3 .

\subsection{AO Imaging}

For the systems with statistically significant trends, we obtained AO imaging data to determine whether these systems had stellar companions that might have caused the observed trend. We identified published AO images (Tanner et al. 2010; Alonso et al. 2014; Howard et al. 2014; Rodriguez et al. 2015; Kraus et al. 2016; Sahlmann et al. 2016; Christiansen et al. 2017; Furlan et al. 2017; Ngo et al. 2017) for all but three of these systems. Of the remaining three systems, two (HD 40307
Table 3

Properties of Outer Gas Giant Companions on Resolved Orbits

\begin{tabular}{lccc}
\hline \hline Companion & Mass $\left(M_{\text {Jup }}\right)$ & $a(\mathrm{au})$ & References \\
\hline Kepler-94 c & $9.84 \pm 0.63$ & $1.60 \pm 0.04$ & Marcy et al. (2014) \\
Kepler-454 c & $4.46 \pm 0.12$ & $1.29 \pm 0.02$ & Gettel et al. (2016) \\
Kepler-68 d & $0.84 \pm 0.05$ & $1.47 \pm 0.03$ & Marcy et al. (2014) \\
Kepler-48 e & $2.07 \pm 0.08$ & $1.85 \pm 0.04$ & Marcy et al. (2014) \\
55 Cnc d & $3.88 \pm 0.07$ & $5.50 \pm 0.03$ & Nelson et al. (2014) \\
GJ 832 b & $0.68 \pm 0.09$ & $3.56 \pm 0.28$ & Wittenmyer et al. (2014) \\
HD 181433 c & 0.65 & 1.76 & Bouchy et al. (2009) \\
HD 181433 d & 0.54 & 3.00 & Bouchy et al. (2009) \\
GJ 676 b & $4.96 \pm 0.96$ & $1.82 \pm 0.06$ & Stassun et al. (2017) \\
WASP-47 c & $1.29 \pm 0.06$ & $1.38 \pm 0.02$ & Sinukoff et al. (2017) \\
\hline
\end{tabular}

and HD 85512) had unpublished archival data obtained with the NACO instrument (Lenzen et al. 2003; Rousset et al. 2003) on the Very Large Telescope (VLT). The HD 40307 data were taken in the $K s$ band with a total integration time of $1.1 \mathrm{hr}$ (ID: 088.C-0832(A); PI: Loehne). The HD 85512 data were obtained in the $K s$ band with a total integration time of 9 minutes (ID: 090.C-0125(A); PI: Mugrauer). Both data sets were obtained without a coronagraph, using a four-point dither pattern.

We downloaded the data for both stars from the ESO archive and processed them using the pipeline outlined in Meshkat et al. (2014). We did not detect any stellar companions in either of these data sets. We show the $5 \sigma K s$ contrast values for both systems in Table 4.

For the remaining system (GJ 3634), we obtained $K_{c}$-band AO images using NIRC2 at Keck on UT 2018 February 5 with an effective integration time of $9 \mathrm{~s}$ and a three-point dither pattern. We identified a close pair of candidate companions at a separation of 1". 8 and used a multipeak point-spread function (PSF) to simultaneously fit GJ 3634 and the two candidate companions in each frame where the companion is resolved. We constructed the PSF as a sum of Moffat and Gaussian functions and fit over a circular aperture of 10 pixels in radius, corresponding to twice the full width at half maximum of the PSF as described in Ngo et al. (2015).

We next integrated the best-fit PSFs for GJ 3634 and its candidate companions over the same aperture to determine their flux ratios. We similarly measured the companion separation and position angle by calculating the difference between the centroids of each star. We then applied the NIRC2 astrometric corrections from Service et al. (2016) to compensate for the NIRC2 array's distortion and rotation. We find that the easternmost candidate companion (labeled as cc1 in Figure 2) has a flux ratio of 116, corresponding to $\Delta K_{c}=5.16$. This companion is separated from GJ 3634 by 1 l" $^{\prime \prime} \pm 0$. !" 002 at a position angle of $177^{\circ} .37 \pm 0.04$ east of north. For the western candidate companion (cc2), we measure a flux ratio of $75 \pm 8$, corresponding to $\Delta K_{c}=4.7 \pm 0.1$. This companion is separated from GJ 3634 by 1 "! $860 \pm 0$ " 002 at a position angle of $203^{\circ} .40 \pm 0.05$ east of north. We calculate our uncertainties as the quadrature sum of the measurement uncertainties and the uncertainty in the distortion solution. However, in one of the three frames, cc1 did not have a regular PSF shape; as a result, we were unable to fit for its peak and location. With just two independent measurements for this companion, we are unable to calculate an empirical measurement uncertainty and therefore only report the astrometric distortion solution uncertainties. 
Table 4

$5 \sigma$ Contrast Curves

\begin{tabular}{|c|c|c|}
\hline System & Separation (arcsec) & $5 \sigma$ Contrast (mag) \\
\hline \multirow[t]{9}{*}{ HD 40307} & 0.79 & 9.47 \\
\hline & 1.90 & 10.87 \\
\hline & 2.99 & 11.72 \\
\hline & 4.07 & 11.45 \\
\hline & 5.16 & 11.91 \\
\hline & 6.24 & 11.90 \\
\hline & 7.33 & 12.06 \\
\hline & 8.42 & 12.63 \\
\hline & 9.50 & 12.66 \\
\hline \multirow[t]{9}{*}{ HD 85512} & 0.79 & 4.41 \\
\hline & 1.28 & 6.95 \\
\hline & 1.76 & 7.40 \\
\hline & 2.25 & 8.06 \\
\hline & 2.74 & 8.68 \\
\hline & 3.23 & 9.34 \\
\hline & 3.69 & 9.95 \\
\hline & 4.18 & 9.44 \\
\hline & 4.67 & 10.07 \\
\hline \multirow[t]{24}{*}{ GJ 3634} & 0.09 & 0.014 \\
\hline & 0.22 & 3.92 \\
\hline & 0.35 & 4.81 \\
\hline & 0.49 & 5.24 \\
\hline & 0.63 & 6.18 \\
\hline & 0.77 & 6.71 \\
\hline & 0.91 & 6.92 \\
\hline & 1.05 & 7.15 \\
\hline & 1.18 & 7.11 \\
\hline & 1.32 & 7.18 \\
\hline & 1.46 & 7.20 \\
\hline & 1.60 & 7.11 \\
\hline & 1.74 & 7.14 \\
\hline & 1.88 & 7.05 \\
\hline & 2.02 & 7.05 \\
\hline & 2.16 & 7.05 \\
\hline & 2.30 & 7.10 \\
\hline & 2.43 & 7.05 \\
\hline & 2.57 & 7.04 \\
\hline & 2.71 & 7.11 \\
\hline & 2.85 & 7.05 \\
\hline & 2.99 & 7.13 \\
\hline & 3.13 & 7.06 \\
\hline & 3.26 & 7.01 \\
\hline
\end{tabular}

For each companion in GJ 3634, we determine stellar masses using PHOENIX spectral models and the Baraffe et al. (1998) zero-age main-sequence models. We first select a PHOENIX model for the primary star based on the published stellar properties and then determine the companion's effective temperature by identifying the PHOENIX model that most closely matches the observed flux ratio. For each PHOENIX model, we determine the corresponding stellar mass and radius using the Baraffe et al. models. For both companions, we find best-fit masses of $0.08 M_{\odot}$. However, with only one epoch of data for this system, it is not possible to determine whether or not these companions are bound to the primary. We note, however, that this is a high proper motion target $(-566.861$, -91.371 $\mathrm{mas} \mathrm{yr}^{-1}$; Gaia Collaboration 2018), and astrometric measurements with just a $1 \mathrm{yr}$ baseline would easily determine whether these companions are bound. We thus examined archival VLT/SPHERE images taken in 2017 May and found the companions at similar positions as in the Keck images from 2018. The difference in relative separation of the companions

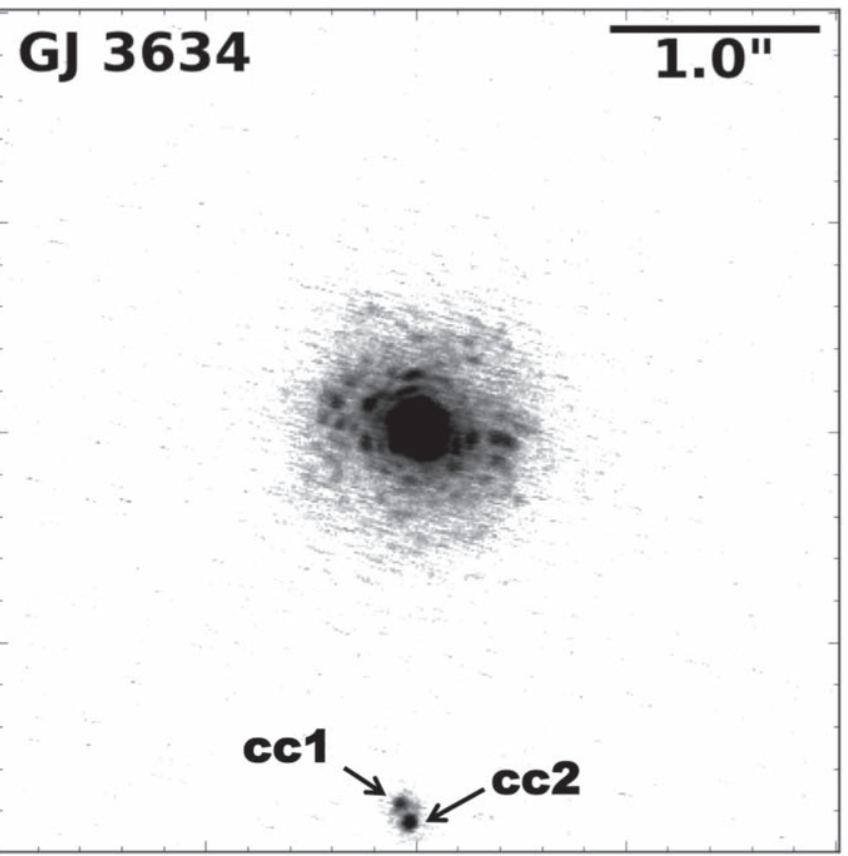

Figure 2. Reduced Keck/NIRC2 $K_{c}$-band image of GJ 3634 showing two candidate companions, labeled cc1 and cc2. Note that the image is shown on a $\log$ scale and is aligned with north toward the top and east toward the left.

between these two epochs is 30 mas for $\mathrm{cc} 1$ and 20 mas for $\mathrm{cc} 2$, which is significantly less than the reported proper motion of GJ 3634, allowing us to rule out the case that the pair of companions are distant background stars.

We next consider whether or not the RV trends in these systems might plausibly be explained by the presence of a nearby stellar companion. Kepler-93, Kepler-97, Kepler-407, and GJ 3634 each have candidate stellar companions, meaning that these systems have one epoch of data showing nearby stars that could be either bound companions or distant background stars. The systems GJ 15A and GJ 676 have confirmed stellar companions that have been shown to have the same proper motion as the primary. We calculate the minimum companion mass in each system needed to explain the observed RV trend using the equation from Torres (1999):

$$
\begin{aligned}
M_{\text {comp }}= & 5.34 \times 10^{-6} M_{\odot}\left(\frac{d}{\mathrm{pc}} \frac{\rho}{\operatorname{arcsec}}\right)^{2} \\
& \times\left|\frac{\dot{v}}{\mathrm{~m} \mathrm{~s}^{-1} \mathrm{yr}^{-1}}\right| F(i, e, \omega, \phi) .
\end{aligned}
$$

In this equation, $d$ is the distance to the star, $\rho$ is the projected separation of the companion and the star on the sky, $\dot{v}$ is the RV trend, and $F(i, e, \omega, \phi)$ is a variable that depends on the orbital parameters of the companion that are currently unconstrained. We use the minimum value of $F, \sqrt{27} / 2$, which corresponds to the minimum companion mass given the other variables of RV slope, distance, and angular separation (Liu et al. 2002). We then compare this minimum mass to the estimated mass of the candidate companion, which we calculate using the measured brightness ratio under the assumption that the candidate companion is located at the same distance as the primary star. We discuss our results for each individual system below. 
Kepler-93 is 96.7 pc away and has a candidate companion with a projected separation of 2!. 29 (Kraus et al. 2016). With an $\mathrm{RV}$ trend of $12.0 \mathrm{~m} \mathrm{~s}^{-1} \mathrm{yr}^{-1}$, this trend corresponds to a minimum companion mass of $8.2 M_{\odot}$. We estimate the mass of the candidate companion using its measured magnitude $M_{K}=5.35$ and assuming an age of $1 \mathrm{Gyr}$. We then use the Baraffe et al. (1998) models to calculate a corresponding mass of $0.57 M_{\odot}$ for this companion. This mass is significantly smaller than the minimum mass needed to explain the RV trend, and we therefore conclude that this candidate companion cannot explain the observed RV trend and keep this system in our sample.

Kepler-97 is 414 pc away and has a candidate companion with a projected separation of 0."385 (Furlan et al. 2017). With an RV trend of $4.5 \mathrm{~m} \mathrm{~s}^{-1} \mathrm{yr}^{-1}$, this trend corresponds to a minimum companion mass of $1.58 M_{\odot}$. The candidate companion in this system has a magnitude $M_{K}=6.28$, corresponding to an estimated companion mass of $0.4 M_{\odot}$ using its estimated age of $8.4 \mathrm{Gyr}$. As this is smaller than the minimum mass needed to explain the RV trend, we leave this system in our sample.

Kepler-407 is 326 pc away and has a candidate companion with a projected separation of 2"' 13 (Kraus et al. 2016). With an $\mathrm{RV}$ trend of $-155.8 \mathrm{~m} \mathrm{~s}^{-1} \mathrm{yr}^{-1}$, this trend corresponds to a minimum companion mass of $1045 M_{\odot}$. Given the companion's measured magnitude of $M_{K}=7.0$ and using its estimated age of $7.5 \mathrm{Gyr}$, the estimated companion mass is $0.3 M_{\odot}$. This is several orders of magnitude smaller than would be required in order to explain the observed RV trend, and we therefore leave this system in the sample.

The system GJ 3634 is 19.8 pc away and has two candidate companions in what appears to be a hierarchical triple system, as discussed above. These two companions are 1."83 away from GJ 3634 and have a mutual separation of 0 ". 087 . The measured RV trend in this system is $9.6 \mathrm{~m} \mathrm{~s}^{-1} \mathrm{yr}^{-1}$, corresponding to a minimum companion mass of $0.018 M_{\odot}$. As discussed earlier, both companions have estimated masses of $0.08 M_{\odot}$, indicating that their combined mass could be responsible for the observed $\mathrm{RV}$ trend. We thus remove this system from our sample in subsequent analyses. We note that the RV trend in this system was previously published in Bonfils et al. (2011). Given their trend, they estimate a minimum mass of $32 M_{\oplus}$ and a minimum period of 200 days. Our AO image is the first to indicate that this trend might be due to the presence of stellar/brown dwarf companions rather than a distant orbiting planet.

The system GJ $15 \mathrm{~A}$ is $3.6 \mathrm{pc}$ away and has a confirmed stellar companion with a projected separation of 20 !" 28 (Howard et al. 2014). With an RV trend of $-0.44 \mathrm{~m} \mathrm{~s}^{-1} \mathrm{yr}^{-1}$, this corresponds to a minimum companion mass of $0.074 M_{\odot}$. The stellar companion in this system has an absolute magnitude of $M_{K}=8.17$, corresponding to an estimated companion mass of $0.175 M_{\odot}$ for an age of $1 \mathrm{Gyr}$. As this estimated companion mass is larger than the minimum companion mass needed to account for the trend, we exclude this system from our subsequent analysis.

Finally, GJ 676 is 15.9 pc away (Gaia Collaboration et al. 2016a, 2016b) and has a confirmed stellar companion at a separation of $47^{\prime \prime}$. With an RV trend of $21.6 \mathrm{~m} \mathrm{~s}^{-1} \mathrm{yr}^{-1}$, this trend corresponds to a minimum companion mass of $167 M_{\odot}$. Given an absolute magnitude of $M_{K}=6.9$, the estimated companion mass is $0.3 M_{\odot}$, assuming an age of $1 \mathrm{Gyr}$. Since this estimated companion mass is well below the minimum mass to account for the observed RV trend, we conclude that this companion could not be producing the observed trend and leave this system in our sample.

\subsection{Trends Due to Stellar Activity}

We next consider whether any of the observed trends might be due to stellar activity. We examined each system in order to determine if the measured RV trend exhibits a correlation with the star's emission in Ca II H \& $\mathrm{K}$ lines as quantified by either the $S_{\mathrm{HK}}$ index or $\log R^{\prime}$ (Wright et al. 2004; Isaacson \& Fischer 2010). We calculated the Spearman rank correlation coefficients between the RV data and this activity indicator after subtracting the orbital solutions for the confirmed inner planets. We considered a correlation coefficient with an absolute value greater than 0.3 to indicate a significant correlation. We find that systems HD 219134, HD 40307, and HD 85512 have significant correlations between stellar activity and the observed RV trend, and we remove these system from our subsequent analysis. We also remove HD 1461 from our analysis, as we determined in Bryan et al. (2016) that this system has a fully resolved long-period signal that is significantly correlated with stellar activity.

There were two systems with RV trends for which we were not able to obtain stellar activity data, including Corot-24 and GJ 3634. We conclude that stellar activity is unlikely to be the cause of the trend in Corot-24, as the amplitude of the observed trend is higher than would be expected for stellar activity signals. Although we cannot determine whether or not the observed RV trend in GJ 3634 might be due to stellar activity, we have already removed this system from further analysis due to the presence of candidate stellar companions that could have caused the observed trend.

After removing systems with either stellar or potentially activity-related sources of RV trends, including HD 219134, HD 85512, HD 40307, HD 1461, GJ 15A, and GJ 3634, we are left with nine systems with statistically significant trends that can plausibly be attributed to the presence of a substellar companion. We plot the RV data for each of these systems after subtracting the orbital solutions for the confirmed inner planets in Figure 3. Trends for GJ 667 C (Anglada-Escudé et al. 2012), GJ 676 (Anglada-Escudé \& Tuomi 2012; Sahlmann et al. 2016), Kepler-93 (Marcy et al. 2014; Dressing et al. 2015), Kepler-97 (Marcy et al. 2014), Kepler-407 (Marcy et al. 2014), and Kepler-454 (Dressing et al. 2015) were previously reported in the published literature.

\subsection{Constraints on Companion Masses and Orbital Semi- major Axes}

We use the RV data to place constraints on the masses and semi-major axes of the long-period companions in each system. The duration and shape of the RV trend places a lower limit on the companion's mass and separation, while the lack of a detection in our AO imaging data places a corresponding upper limit on these quantities. As described in Bryan et al. (2016), we calculate two-dimensional probability distributions for each companion using an equally spaced $50 \times 50$ grid in logarithmic mass (true mass, not $m$ sin $i$ ) and a logarithmic semi-major axis spanning a range of 0.3-500 $M_{\text {Jup }}$ and 0.5-500 au. In each grid cell, we inject 500 simulated companions and determine whether or not they are consistent with the RV observations as 

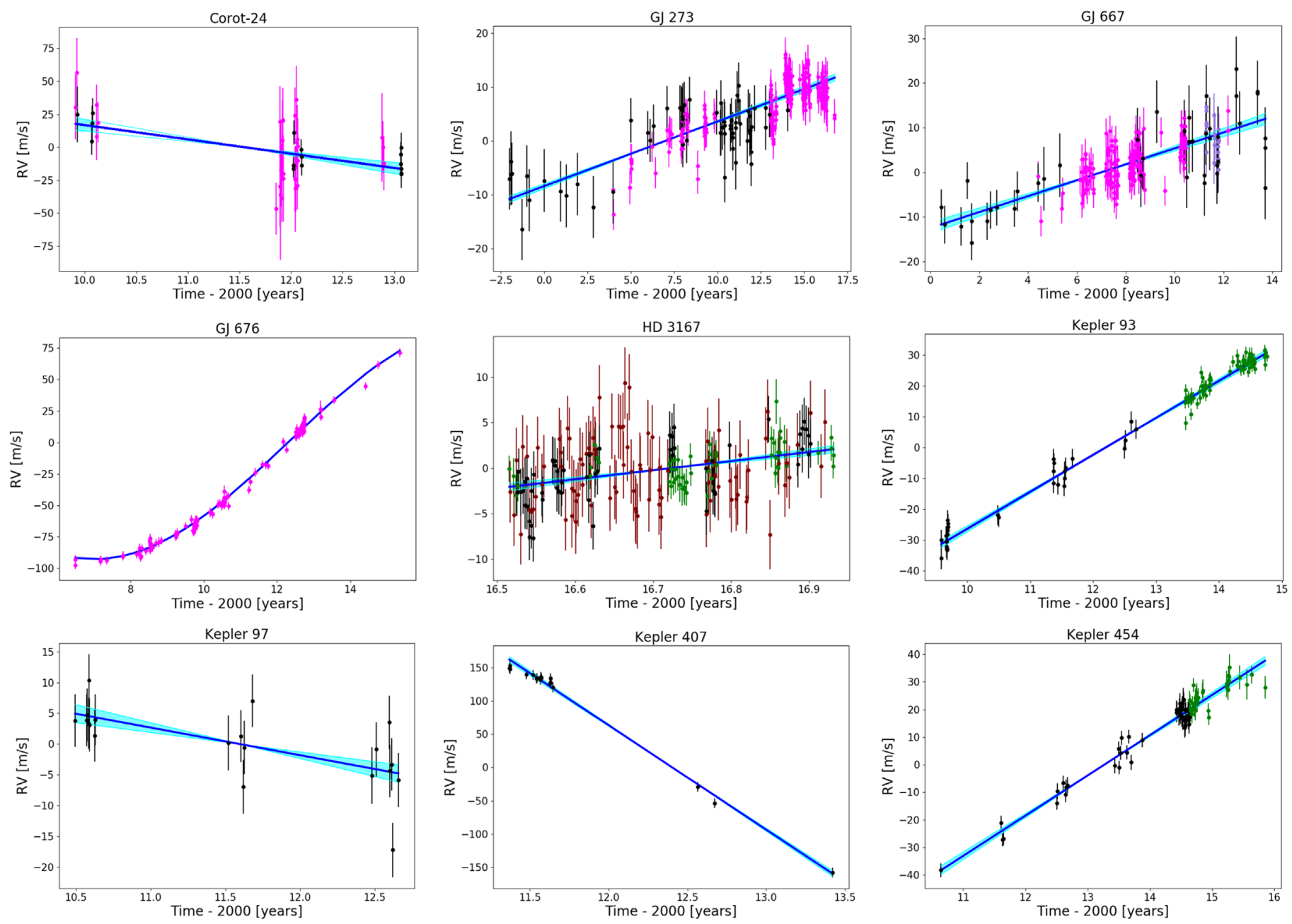

Figure 3. Best-fit accelerations to the RV data with a $3 \sigma$ trend. The best-fit trend is shown as a solid blue line, and the $1 \sigma$ errors on the slope are presented as light blue shaded regions. The different colored data points represent RVs taken using different telescopes: black $=$ HIRES, green $=$ HARPS-N, pink $=$ HARPS, light purple $=$ PFS, and maroon $=$ APF. Note that GJ 676 has a curved trend, which allows us to place much tighter constraints on the mass and separation of the companion producing that trend.

follows. We first draw a set of orbital parameters for the confirmed inner planets from the previous MCMC fits, and then we subtract away this orbital solution to preserve any long-term trend signal. We then draw a mass and semi-major axis value from within the grid box from a uniform distribution in $\log (M)$ and $\log (a)$ and an inclination from a uniform distribution in $\cos i$. We draw our eccentricity values from a beta distribution with $a=1.12$ and $b=3.09$, which are derived from a fit to the population of long-period gas giant planets from RV surveys (Kipping 2013). Given a fixed semimajor axis, mass, and eccentricity for each simulated companion, we then fit for the remaining orbital parameters, including time of periastron, argument of periastron, and velocity zero-point, and calculate the corresponding loglikelihood value of the best-fit solution.

After repeating this process 500 times in each grid cell, we convert the resulting $50 \times 50 \times 500$ cube of log-likelihood values to probabilities and marginalize over our 500 samples in each grid cell to yield a two-dimensional probability distribution in mass and semi-major axis for each system. We calculate two-dimensional probability distributions for all systems in our sample, regardless of whether or not they have statistically significant trends. The only difference between those systems with and without trends is that we use our AO imaging data to place an upper limit on the companion mass and semi-major axis in the trend systems as discussed in Bryan et al. (2016). We note that for GJ 273, as a result of its close distance (3.8 pc) and the limited angular extent of the available contrast curve, the contrast curve for this system does not provide significant constraints on the mass and separation of the companion where the probability density for the companion is large. Similarly, for Corot-24, due to the significant distance of the system $(600 \mathrm{pc})$, constraints provided by the available contrast curve at smaller separations where the probability density for the companion is high are not significant.

Figure 4 shows the posterior distributions for the systems with $3 \sigma$ trends, while Table 5 indicates the corresponding $1 \sigma$ limits in mass and semi-major axis for each companion.

\subsection{Completeness Maps}

We evaluate our sensitivity to distant companions in each system by calculating the completeness as a function of mass and orbital semi-major axis after taking into account the time baseline, number of data points, and measurement errors for each data set. As before, we start with a $50 \times 50$ grid in mass and semi-major axis evenly spaced in log space from 0.3 to $500 M_{\text {Jup }}$ and 0.5 to $500 \mathrm{au}$. For each grid box, we inject 500 

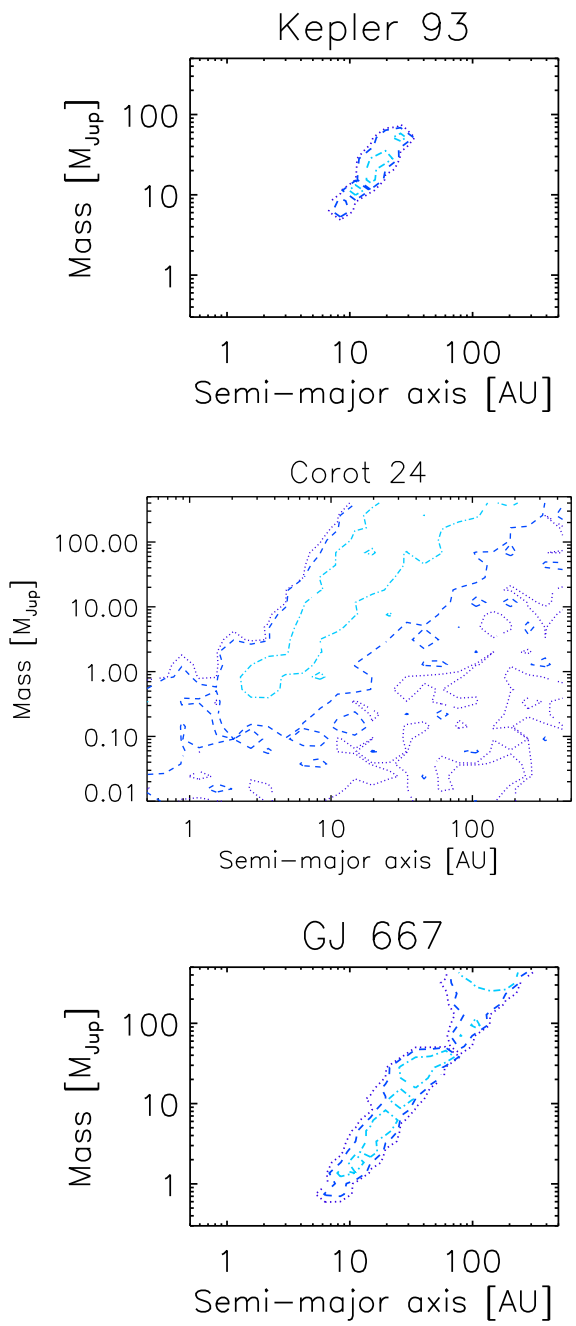
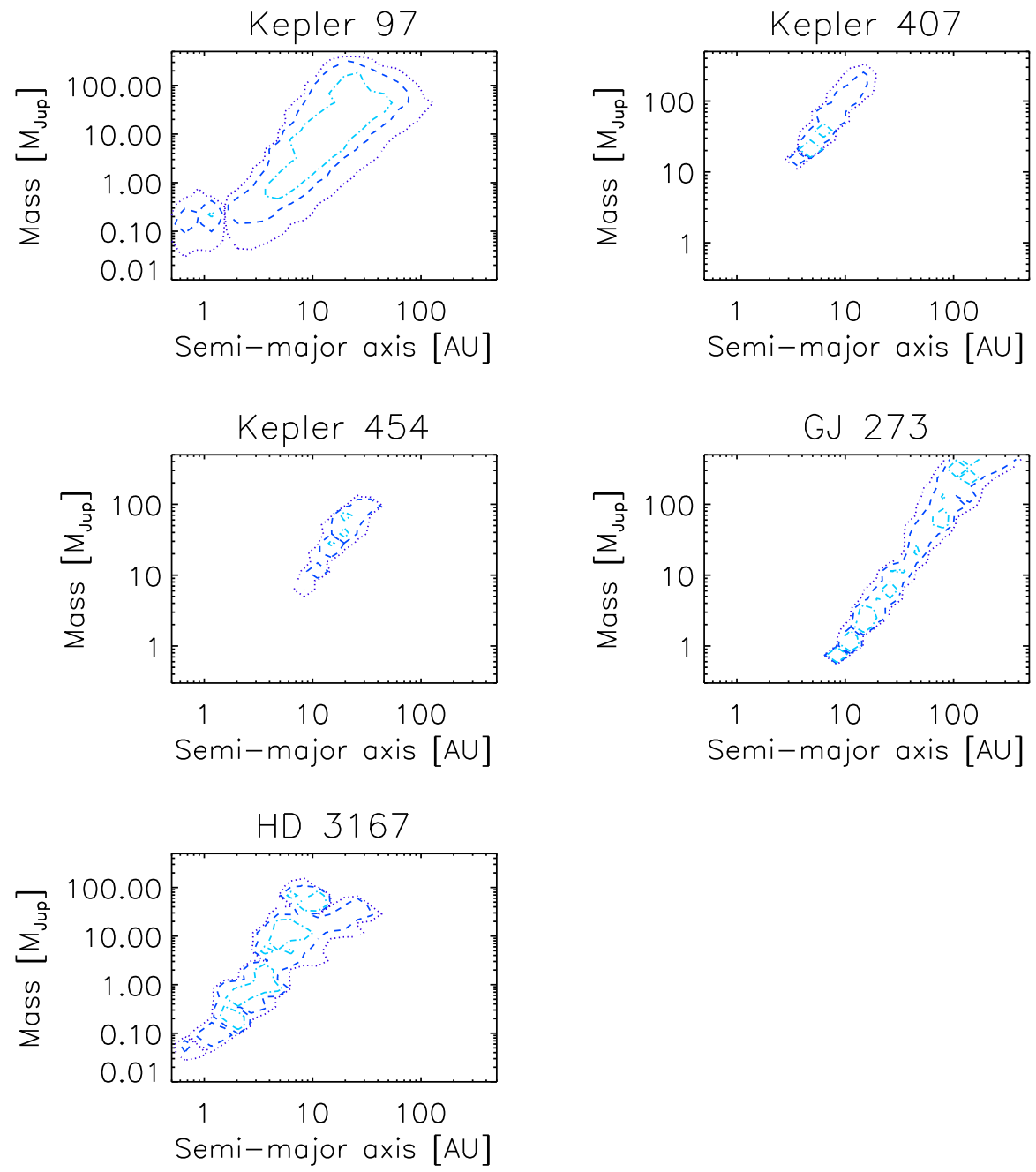

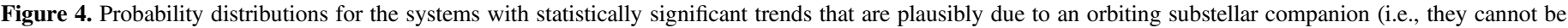

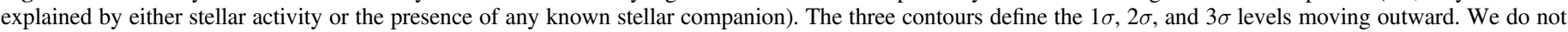
show the probability distribution for GJ 676 here, as the probability density is concentrated in just a few grid points and the contours are therefore unresolved.

Table 5

Constraints on Companion Properties

\begin{tabular}{lcc}
\hline \hline Companion & Mass $\left(M_{\text {Jup }}\right)$ & Semi-major Axis (au) \\
\hline Kepler-93 c & $11.3-51.6$ & $9.6-25.9$ \\
Kepler-97 c & $0.18-166$ & $1.2-60.3$ \\
Kepler-407 c & $11.4-51.6$ & $3.1-7.3$ \\
Corot-24 d & $0.27-401$ & $0.5-186$ \\
Kepler-454 d & $7.2-81.3$ & $9.6-29.8$ \\
GJ 273 d & $0.55-430$ & $7.3-214$ \\
GJ 667 h & $1.2-430$ & $8.4-214$ \\
HD 3167 e & $0.05-85$ & $0.8-22$
\end{tabular}

simulated companions, where we draw a mass and semi-major axis from a uniformly spaced distribution across each grid box, an eccentricity value from the $\beta$ distribution, an inclination from a uniform distribution in $\cos i$, and the remaining orbital elements from uniform distributions. We then calculate the RV signal from this simulated companion at each observation epoch. We add noise into these simulated RVs by drawing from a Gaussian distribution with a width defined by $\sqrt{\sigma_{i}^{2}+\sigma_{\text {jit }}^{2}}$, where $\sigma_{i}$ is the instrumental uncertainty (randomly shuffled from the original data set) and $\sigma_{\mathrm{jit}}$ is the stellar jitter estimated from the earlier MCMC fits. To assess whether a simulated planet would be detected, we fit each simulated set of RVs with a one-planet orbital solution, a linear trend, and a flat line. We compared these model fits using the BIC (Kass \& Raftery 1995) in order to determine the simplest model that can provide an adequate fit to the data. If the BIC values for either the one-planet model fit or the linear trend were smaller than the BIC value for the flat line by at least 10 , we concluded that the simulated planet would have been detected. However, if the flat line was preferred or the difference in BIC was less than 10 , we counted this as a nondetection. We repeated this process for each simulated companion injected into each grid box, using our "detected/not detected" determinations to calculate the completeness over the entire grid.

Perhaps unsurprisingly, we find that the average sensitivity to companions in systems with super-Earths discovered via the transit method is significantly less than in systems with RVdetected super-Earths. This likely reflects the substantially greater investment of RV time required to detect a planet with an unknown orbital period and phase versus the transit case, where these two quantities are known precisely in advance. The $\mathrm{RV}$-only detections must also achieve a higher significance in their measurement of the RV semi-amplitude in order to be considered a secure detection (see representative trend system 

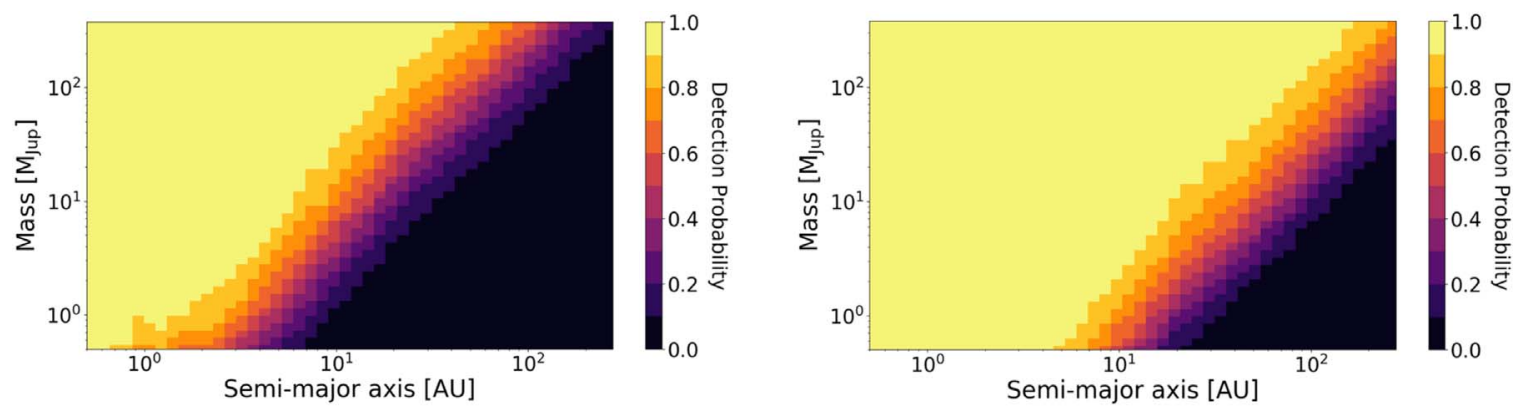

Figure 5. Sensitivity maps for the systems with super-Earths discovered using the transit method (left) and RV method (right). The RV detections typically require much more extensive data sets and have longer baselines than observations of transiting planet systems, resulting in different levels of completeness for these two samples.

GJ 273; Forveille et al. 2009; Butler et al. 2017), whereas for $\mathrm{RV}$ follow-up of transiting planets, even marginally significant measurements of this quantity still provide useful constraints on the planet density (see representative trend system Kepler97; Marcy et al. 2014). We show the resulting completeness maps in Figure 5, with systems discovered using the transit method plotted separately from systems discovered using RVs in order to illustrate the different average sensitivities of these two samples.

\section{Discussion}

\subsection{The Occurrence Rate of Gas Giant Companions}

In this section, we utilize our probability distributions (see Section 3.4) for each system to determine the underlying distribution and corresponding occurrence rate for the observed population of long-period gas giant companions in these systems. We follow the methodology laid out in Bryan et al. (2016) and present a summary of the steps here. We first assume that this population of companions is distributed in mass and semi-major axis space according to a double power law of the form $f(m, a)=C m^{\alpha} a^{\beta}$ (e.g., Tabachnik \& Tremaine 2002; Cumming et al. 2008). The likelihood for a set of $N$ exoplanet systems is given by

$$
\mathscr{L}=\Pi_{i=1}^{N} p\left(d_{i} \mid C, \alpha, \beta\right),
$$

where $p\left(d_{i} \mid C, \alpha, \beta\right)$ is the probability of the RV data set given the power-law coefficients $C, \alpha$, and $\beta$. Assuming that each system has at most one outer companion, this likelihood is then the sum of the probability that a given system contains one planet and the probability that the system contains zero planets. The probability of a system containing zero planets is given by

$$
p\left(d_{i}, 0 \mid C, \alpha, \beta\right)=p\left(d_{i} \mid 0\right)[1-Z],
$$

where $Z$ is the probability that the system contains a planet within a range of masses and semi-major axes (determined by integrating the power-law distribution over the specified range), and $p\left(d_{i} \mid 0\right)$ is the probability of obtaining the RV data set given that there is no planet in the system.

The probability of a system having one companion given their distribution in mass and semi-major axis space is

$$
\begin{aligned}
p\left(d_{i}, 1 \mid C, \alpha, \beta\right)= & \int_{a_{1}}^{a_{2}} d \ln a \int_{m_{1}}^{m_{2}} d \ln m \\
& \times p\left(d_{i} \mid a, m\right) C m^{\alpha} a^{\beta},
\end{aligned}
$$

where $p\left(d_{i} \mid a, m\right)$ is the probability of a companion being located at a given mass and semi-major axis, which we know from our previously determined probability distributions (see Section 3.4). To determine the likelihood of a given set of $C, \alpha$, and $\beta$ given our RV data sets, we combine the probabilities of a system having one planet and a system having zero planets as follows:

$$
\mathscr{L}=\Pi_{i=1}^{N}\left[p_{i}\left(d_{i}, 0 \mid C, \alpha, \beta\right)+p_{i}\left(d_{i}, 1 \mid C, \alpha, \beta\right)\right] .
$$

As in Bryan et al. (2016), we incorporate the probability distributions for all systems in this framework, not just the distributions for systems that have statistically significant trends. This allows us to treat all systems consistently, regardless of whether or not they have a statistically significant trend. Phrased another way, this allows for the possibility of marginal trend detections, rather than assuming a binary classification system in which any star with a less than $3 \sigma$ trend is counted as a nondetection. For the nine systems hosting exterior gas giant $\left(>0.5 M_{\text {Jup }}\right.$ ) companions on resolved orbits outside 1 au, we replace the probability distributions calculated from the RV trends with ones where the probability density is concentrated in a single grid point closest to the best-fit mass and orbital separation of the resolved companion (see Table 3 for these values).

Two of these systems (GJ 676 and Kepler-454) have both statistically significant trends and resolved gas giant companions, while HD 181433 has two resolved gas giant planet companions. In these cases, we select the innermost gas giant planet for inclusion in our power-law fit. We also note that our choice to use the inner versus outer companion in the system with more than one gas giant companion does not impact our derived power-law coefficients or occurrence rates.

We determine the values of $C, \alpha$, and $\beta$ that maximize the value of $\mathscr{L}$ by first performing a grid search where we vary each of these power-law coefficients and then carrying out an MCMC fit initialized near the location of the optimal grid point. Because these parameters are both poorly constrained and highly correlated, we find that the use of a preliminary grid search allows us to reliably identify the global maximum and reduces the convergence time in our MCMC chains.

We next use the results of these power-law fits to calculate an integrated occurrence rate for the observed population of gas giant companions over a range of masses and semi-major axes. We first calculate the integrated companion frequency separately for systems discovered using the transit method versus the RV method. Given the significant differences in completeness for these two samples of systems, this allows us 
Table 6

Total Occurrence Rates for Companions

\begin{tabular}{lccc}
\hline \hline & $1-10 \mathrm{au}$ & $1-20 \mathrm{au}$ & $1-50 \mathrm{au}$ \\
\hline $0.5-20 M_{\text {Jup }}$ & $38 \% \pm 7 \%$ & $39 \% \pm 7 \%$ & $41_{-7}^{+8} \%$ \\
$0.5-13 M_{\text {Jup }}$ & $36_{-6}^{+7} \%$ & $41_{-7}^{+8} \%$ & $40_{-7}^{+8} \%$ \\
$1-20 M_{\text {Jup }}$ & $35 \% \pm 7 \%$ & $35 \% \pm 7 \%$ & $38_{-7}^{+8} \%$ \\
$1-13 M_{\text {Jup }}$ & $34 \% \pm 7 \%$ & $38 \% \pm 7 \%$ & $39_{-7}^{+8} \%$ \\
\hline
\end{tabular}

to evaluate the degree to which these sensitivities impact the integrated occurrence rates. We ran the grid search and MCMC analysis of each sample separately. When we calculated the occurrence rates for these two samples of systems over a mass range of $0.5-20 M_{\text {Jup }}$ and a semi-major axis range of 1-20 au, we found that the occurrence rate of companions in the transiting planet sample is $41_{-10}^{+10} \%$, while the occurrence rate of the RV planet sample is $34_{-10}^{+11} \%$. These two values are consistent at the $0.5 \sigma$ level. We note that the uncertainties on these occurrence rates are dominated by the number of systems in each sample, which are similar (34 for the transiting planet sample, 25 for the RV sample).

We next calculate the frequency of companions for the combined sample over different ranges in mass and semi-major axis in order to assess how occurrence rates depend on our chosen integration ranges. Table 6 shows the resulting occurrence rates for the combined sample. We note that, as in Bryan et al. (2016), the values for the power-law coefficients $\alpha$ and $\beta$ vary significantly depending on our chosen integration range as a result of the poorly constrained companion masses and separations in these systems. However, we find that we obtain consistent results for the integrated occurrence rate for these companions across a wide range of integration ranges. This is because the strongest constraint we obtain from these data is the total number of companions in these systems, while their locations are poorly constrained. As a result, we find that the preferred values for $C, \alpha$, and $\beta$ in our fits are correlated in a way that preserves the total number of companions regardless of the fitting range used. This stands in contrast to studies examining populations of planets with tightly constrained masses and orbital semi-major axes (e.g., Cumming et al. 2008; Bowler et al. 2010), where the values of $\alpha$ and $\beta$ are much better constrained by the data. For these systems, we would expect the integrated occurrence rate to rise as we increase the range in mass and semi-major axis, reflecting our much better knowledge of the planet occurrence rate density. This is an important point to consider when comparing our occurrence rate to those from surveys focusing on planets with fully resolved orbits, as we will discuss below.

Our estimated occurrence rate has a relatively weak dependence on the assumed eccentricity distribution. As discussed in Section 3.4, we draw eccentricities from a beta distribution to calculate the probability distributions for each companion. When we recalculated our probability distributions using either circular orbits or a uniform distribution in eccentricity, we found occurrence rates of $36 \% \pm 7 \%$ and $40 \% \pm 7 \%$, respectively. Both of these values are consistent with our nominal occurrence rate of $39 \% \pm 7 \%$ calculated using the beta distribution. We also test our assumptions regarding the inclination distribution of the companions. Previous dynamical studies have shown that for some multitransiting systems, an exterior companion must have a similar inclination (i.e., Becker \& Adams 2017). We test an extreme version of this scenario by recalculating all probability distributions for multitransiting systems using a restricted inclination range of $5^{\circ}\left(87^{\circ} .5-92^{\circ} .5\right)$. We rerun the occurrence rate calculation for all systems using these restricted inclination distributions for the multitransiting systems and obtain an occurrence rate of $38 \% \pm 7 \%$. We conclude that restricting the inclination for these systems does not significantly impact the occurrence rate. Finally, we explore how our calculated occurrence rate might be impacted if some of the less significant trends are caused by stellar activity. We test an extreme scenario in which we assume that all systems with $<1 \sigma$ trends (15 systems) are caused by stellar activity by replacing these trends with flat lines. We find that when we recalculate the occurrence rate over the range $0.5-20 M_{\text {Jup }}$ and $1-20 \mathrm{au}$, it is $37 \% \pm 7 \%$, consistent with the original occurrence rate of $39 \% \pm 7 \%$. We take this a step further and replace all systems with $<2 \sigma$ trends (40 systems) with flat lines. When we rerun the occurrence rate calculation, we find an occurrence rate of $30 \%(+7 \%-6 \%)$ over the range 0.5-20 $M_{\text {Jup }}$ and 1-20 au. Thus, the bulk of the occurrence rate is being driven by stronger trends and resolved companions, as expected, and we conclude that it is unlikely that stellar activity is the cause of a majority of these weaker trends.

\subsection{Comparison to Field Jupiter Analog Occurrence Rates}

We now aim to determine whether the rate of gas giant companions in super-Earth systems is higher or lower than the average occurrence rate for Sun-like field stars. If there is no correlation (positive or negative) between the presence of an inner super-Earth and an outer gas giant companion, we would expect these two rates to be consistent with each other.

In this study, we examine 65 super-Earth-hosting stars, where we define a super-Earth as a planet having either a mass between 1 and $10 M_{\oplus}$ or a radius between 1 and $4 R_{\oplus}$, depending on the detection method. Similarly, we define a long-period gas giant as a planet with a mass between 0.5 and $20 M_{\text {Jup }}$ and semi-major axis between 1 and $20 \mathrm{au}$. When comparing to previous work, we vary this definition of longperiod gas giant planet to better match individual surveys, as discussed below.

There have been several studies that have sought to quantify the frequency of long-period gas giant planets, including Foreman-Mackey et al. (2016), Rowan et al. (2016), and Wittenmyer et al. (2016). Wittenmyer et al. (2016) calculated the occurrence rate of Jupiter analogs over the range $0.3-13 M_{\text {Jup }}$ and between 3 and 7 au for a sample of 202 stars observed as a part of the $17 \mathrm{yr}$ Anglo-Australian Planet Search. For their sample of targets, they only considered planets with fully resolved orbits and found an integrated occurrence rate of $6.2_{-1.6}^{+2.8} \%$ over this range, assuming binomial statistics (i.e., they did not fit a power-law distribution). Integrating our sample over this same mass and semi-major axis range, we find an occurrence rate of $34 \% \pm 7 \%$, which differs from the Wittenmyer et al. (2016) value by $3.7 \sigma$ (Figure 6 ). We note that for this occurrence rate calculation, in a system with two gas giant companions (either both resolved or one resolved and one statistically significant trend), if the innermost resolved companion does not fall within this integration range, we use the outer companion (this was the case for systems HD 181433, GJ 676, and Kepler-454). As stated previously, the inclusion of either the closer resolved gas giant companions or the farther 


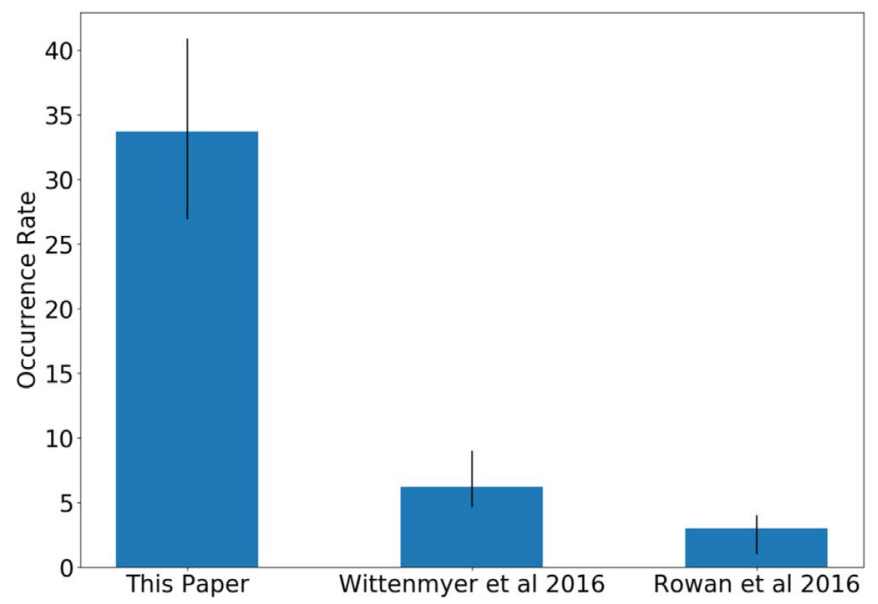

Figure 6. Compared to the Jupiter analog occurrence rate estimates published in Rowan et al. (2016) and Wittenmyer et al. (2016), this study finds a higher occurrence rate of distant gas giant planets in super-Earth systems than would be expected just based on chance. Occurrence rate integration ranges are $0.3-13 M_{\text {Jup }}$ and 3-7 au for this paper and Wittenmyer et al. (2016) and 0.3-3 $M_{\text {Jup }}$ and 3-6 au for Rowan et al. (2016).

out signals does not affect our occurrence rate calculations over the range $0.5-20 M_{\text {Jup }}$ and 1-20 au.

In order to determine whether or not the difference between our occurrence rate of $34 \% \pm 7 \%$ and the $6.2_{-1.6}^{+2.8} \%$ rate is meaningful, we must consider the possible biases introduced by our decision to consider trends rather than limit our study to companions with fully resolved orbits. Specifically, our occurrence rate is partially derived from a population of planets with probability distributions extending over a wide range of masses and semi-major axes. This means that when we integrate over the relatively narrow range used in the Wittenmyer study, our occurrence rate may be inflated by the inclusion of planets whose probability distributions overlap with this integration range, even though the planets themselves are in fact located on more distant orbits.

To quantify this effect, we assume that planets in our sample are distributed according to a negative power law in mass and a flat power law in semi-major axis. Specifically, we adopt the $\alpha$ value of -0.31 from Cumming et al. (2008) and a $\beta$ value of 0 . We choose not to adopt the Cumming et al. power-law coefficient for semi-major axis $(\beta=0.26)$, as this coefficient was derived from a fit to the population of gas giant planets inside $3 \mathrm{au}$. This fit indicates that the frequency of these planets rises with increasing semi-major axis, but this is inconsistent with current constraints from both RV and direct-imaging surveys (Bowler 2016; Bryan et al. 2016; Clanton \& Gaudi 2016; Bowler \& Nielsen 2018), which prefer much flatter distributions at large semi-major axes. In Bryan et al. (2016), we found that for the population of gas giant planets with longperiod companions, the occurrence rate of these companions decreases with increasing semi-major axis. While the current small sample size of directly imaged planets makes it difficult to determine their mass and semi-major axis distribution, their overall low occurrence rate indicates that a rising power law in semi-major axis is likely not applicable at wide separations.

For each of the nine systems with a statistically significant trend that do not have a resolved companion in this mass and semi-major axis range, we draw from the $\alpha=-0.31, \beta=0$ power-law distribution until we have generated a sample of 100 simulated planets with a cutoff mass of $20 M_{\text {Jup }}$ that lie within the favored region of mass/semi-major axis parameter space where the probability of there existing a planet given the RV trend is greater than the probability of there being no planet given the RV trend. For each system, we then count the fraction of planets that fall within the range $3-7$ au and $0.3-13 M_{\text {Jup }}$. For the resolved companions, we count three companions that fall within this range and were thus included in the occurrence rate calculation. Averaging across all of the trend and resolved companion systems, we find that $61 \%$ of our simulated planet population lies inside this range. If we rescale our occurrence rate to account for the fact that $\sim 2 / 5$ of our occurrence rate might be attributed to companions outside the 3-7 au semimajor axis range, we would then derive a corrected occurrence rate of $21 \% \pm 4 \%$ for our sample over this semi-major axis range. This reduced occurrence rate is still inconsistent with the Wittenmyer et al. value at the $3 \sigma$ level.

We note that Wittenmyer et al. (2016) mentioned that they found 45 trends in their sample of systems. However, these trends could not be Jupiter analogs located between 3 and 7 au because the $8 \mathrm{yr}$ minimum baseline (the specified cutoff for system inclusion in the 202 star sample) would have been sufficient to determine whether or not the orbit was within 3-7 au (R. A. Wittenmyer 2018, private communication). Furthermore, while 8 yrs was the nominal cutoff, over $90 \%$ of systems had baselines above 4000 days ( $11 \mathrm{yr})$, while a majority of the sample had baselines over 6000 days ( $\sim 16.5 \mathrm{yr})$. We therefore conclude that Wittenmyer et al.'s decision to ignore trends in their occurrence rate calculation is unlikely to result in systematic differences in sensitivity as compared to our study.

We next consider results from other studies that provide independent estimates of the frequency of long-period gas giant planets around nearby stars. Rowan et al. (2016) estimated the occurrence rate of Jupiter analogs using a sample of 1122 stars, where they defined a Jupiter analog as a planet with a mass between 0.3 and $3 M_{\text {Jup }}$ and semi-major axis between 3 and 6 au. As with Wittenmyer et al., they only considered planet detections with fully resolved orbits in their analysis. Over this range, they found an occurrence rate of $1 \%-4 \%$. While this integration range has a relatively strict mass limit, we note that previous power-law fits to the population of RV-detected planets consistently indicate that lower-mass gas giants are more common than higher-mass gas giants (Cumming et al. 2008; Bryan et al. 2016), suggesting that their upper bound of $3 M_{\text {Jup }}$ versus our upper bound of $13 M_{\text {Jup }}$ is unlikely to change this integrated occurrence rate very much. We therefore conclude that our occurrence rate is likely higher than the rate from this study as well, with the same caveats as for the Wittenmyer et al. comparison.

For our last comparison, we turn to Foreman-Mackey et al. (2016), who calculated the frequency of long-period planets between 1.5 and 9 au and 0.01 and $20 M_{\text {Jup }}$ using transit detections from the Kepler photometry. Unlike the previous two RV studies, a majority of the long-period planets in their sample have just one observed transit. Although this study is able to place some loose constraints on the orbital periods of these planets based on their measured transit durations, these constraints are nearly as broad as those for our RV trend systems. For this parameter space, Foreman-Mackey et al. (2016) found an occurrence rate density of $0.068 \pm 0.019$, corresponding to an integrated occurrence rate of $92.5 \% \pm$ $25.7 \%$. Over a similar semi-major axis range and a more 
limited mass range (1-10 au and $\left.0.5-20 M_{\mathrm{Jup}}\right)$, we find an occurrence rate density of $0.045 \pm 0.009$ and an integrated occurrence rate of $38 \% \pm 7 \%$. While these two occurrence rate densities are formally consistent, three-quarters of ForemanMackey et al.'s (2016) sample consists of planets whose estimated masses are less than $0.2 M_{\text {Jup }}$, whereas all of our candidate companions have minimum masses higher than this threshold. We therefore conclude that there is relatively little overlap between the two planet samples, making this comparison less relevant than the studies by Rowan et al. (2016) and Wittenmyer et al. (2016).

\subsection{Frequency of Super-Earths in Jupiter Analog Systems}

Now that we have determined the frequency of long-period gas giants in super-Earth systems, we can ask what fraction of the long-period gas giants orbiting field stars are drawn from this population (i.e., what fraction of long-period gas giants have inner super-Earths?). We can express this as a conditional probability,

$$
p(\mathrm{SE} \mid \mathrm{LPG})=\frac{p(\mathrm{LPG} \mid \mathrm{SE}) \times p(\mathrm{SE})}{p(\mathrm{LPG})},
$$

where $p(\mathrm{SE})$ is the probability that a given star hosts a superEarth and $p(\mathrm{LPG})$ is the probability of hosting a long-period gas giant planet. We note that Zhu \& Wu (2018) presented a similar equation and provided an independent estimate of this probability, which we discuss in more detail below. If we take our estimate of the frequency of Jupiter analogs in super-Earth systems, we can use this equation to calculate what fraction of Jupiter analogs host inner super-Earths. For consistency, we integrate the conditional occurrence rate $P(\mathrm{LPG} \mid \mathrm{SE})$ from our study over the same mass and semi-major axis range as Wittenmyer et al. (2016), resulting in $P(\mathrm{LPG} \mid \mathrm{SE})=$ $34 \% \pm 7 \%$ and $P(\mathrm{LPG})=6.2_{-1.6}^{+2.8} \%$. We take the super-Earth occurrence rate of $P(\mathrm{SE})=30 \% \pm 3 \%$ from $\mathrm{Zhu}$ et al. (2018b). Using Equation (7), we find that the conditional probability that there is at least one super-Earth in a system hosting a Jupiter analog is $P(\mathrm{SE} \mid \mathrm{LPG})=164_{-83}^{+57} \%$. Although our definition for this quantity disallows values greater than $100 \%$, it is likely that some of the trends that we include in our Jupiter analog occurrence rate calculation may be caused by planets beyond 7 au. As discussed in Section 4.2, accounting for this correction can reduce our Jupiter analog occurrence to $21 \% \pm 4 \%$ if we assume a flat power-law distribution in semimajor axis. Using this lower value in the conditional probability calculation, we find $P(\mathrm{SE} \mid \mathrm{LPG})=102_{-51}^{+34} \%$. Given the relatively large uncertainties on both the occurrence rates used in Equation (7) and the estimated semi-major axis distribution of planets at these large separations, it is difficult to obtain a precise constraint on the occurrence of super-Earths in Jupiter analog systems, $P(\mathrm{SE} \mid \mathrm{LPG})$. Nevertheless, this result does appear to suggest that most, if not all, systems hosting a Jupiter analog also host one or more inner super-Earths.

Zhu \& Wu (2018) recently published an independent estimate of the occurrence rate of gas giant companions in super-Earth systems, $P(\mathrm{LPG} \mid \mathrm{SE})$, as well as the conditional occurrence rate of super-Earths in Jupiter analog systems, $P$ (SE $\mid$ LPG). They also found that super-Earth systems host more cold Jupiters than would be expected based on chance alone and concluded that most, if not all, systems hosting cold Jupiters also host inner super-Earths. Zhu \& Wu (2018) estimated $P(\mathrm{LPG} \mid \mathrm{SE})$ using a sample of 22 transiting superEarth systems from Kepler with radii in the range $1-4 R_{\oplus}$ and $32 \mathrm{RV}$ systems with masses $<20 M_{\oplus}$. Assessing the overlap between our two samples, we include 21 of their 22 transiting planet systems in our study and 10 of their RV systems. While we missed Kepler-89 in our study, for our RV sample, only 10 of the RV systems in Zhu \& Wu (2018) meet our requirement that $m \sin i$ be less than $10 M_{\oplus}$. For the purpose of this calculation, Zhu \& Wu (2018) defined cold Jupiters as planets with $m \sin i>0.3 M_{\mathrm{Jup}}$ and orbital periods $>1 \mathrm{yr}$ and counted the number of cold Jupiters in these systems as reported in the NASA Exoplanet Archive. This results in an occurrence rate of $P(\mathrm{LPG} \mid \mathrm{SE})=32 \% \pm 8 \%$, assuming $100 \%$ completeness. Using an equivalent conditional probability to our Equation (7), Zhu \& Wu $(2018)$ set $P(\mathrm{LPG})=10 \%$ and $P(\mathrm{SE})=30 \%$ and concluded that $P(\mathrm{SE} \mid \mathrm{LPG})=90 \% \pm 20 \%$.

It is reassuring that $\mathrm{Zhu} \& \mathrm{Wu}$ 's estimated values for $P(\mathrm{LPG}$ $\mathrm{SE})$ and $P(\mathrm{SE} \mid \mathrm{LPG})$ are consistent with our values, despite differences in both sample selection and methodology. However, we note that more quantitative comparisons are difficult given that the authors of this study did not define an explicit upper boundary in mass and semi-major axis for their cold Jupiter sample. Our results demonstrate that the sensitivity of these RV data sets to cold Jupiters can vary greatly from system to system as a result of differences in baseline, cadence, and sample size. Without an upper boundary in semi-major axis, it is difficult to evaluate whether or not Zhu \& Wu's implicit assumption of $100 \%$ completeness is valid for their chosen sample. Similarly, if we wish to compare our value for $P(\mathrm{LPG} \mid \mathrm{SE})$, which requires integration over a finite range in mass and semi-major axis space, to that of $\mathrm{Zhu} \& \mathrm{Wu}$, it is unclear what limits of integration we should use.

As noted above, our study also uses a different mass cutoff for our RV super-Earth sample selection than that utilized by $\mathrm{Zhu} \& \mathrm{Wu}$. While both studies use the same radius range for the transiting planet sample, we require our RV super-Earths to have $m \sin i$ between 1 and $10 M_{\oplus}$, while Zhu \& Wu (2018) used a more generous range of $1-20 M_{\oplus}$. As a result, $40 \%$ of their 54 super-Earth systems contain planets with $m$ $\sin i>10 M_{\oplus}$. This alternate definition increases the likelihood that their sample contains a substantial fraction of Neptunemass planets (see Section 2 for a discussion of this probability for our RV sample). However, while this is an important difference, the fact that we obtain consistent estimated values for $P(\mathrm{LPG} \mid \mathrm{SE})$ in both studies suggests that this choice may not substantially alter their conclusions.

\subsection{Implications of Our Results for Super-Earth Formation and Migration Models}

Although it is difficult to make quantitative comparisons without a better understanding of the power-law distribution for the long-period gas giant planets in our sample, our results indicate that there is a higher occurrence rate for gas giants in systems hosting inner super-Earths than for field stars. Furthermore, if we take our integrated occurrence rate of $34 \% \pm 7 \%$ between 3 and 7 au and 0.3 and $13 M_{\text {Jup }}$ at face value, as well as the overall occurrence rates of super-Earths and long-period gas giant planets (Howard et al. 2010; Fressin et al. 2013; Petigura et al. 2013; Rowan et al. 2016; Wittenmyer et al. 2016; Zhu et al. 2018a), Equation (7) would 
suggest that a significant majority of long-period gas giant planets have inner super-Earths.

\subsubsection{Long-period Gas Giants Do Not Hinder Super-Earth Formation, Did Not Migrate Large Distances}

The apparent correlation between the occurrence of inner super-Earths and outer gas giants suggests that gas giant companions do not hinder super-Earth formation by cutting off the flow of solids to the inner disk, stirring up the velocity distribution of these solids, or preventing super-Earths formed at larger separations from migrating inward (Walsh et al. 2011; Batygin \& Laughlin 2015; Izidoro et al. 2015; Moriarty \& Fischer 2015). We also note that four of the super-Earth systems in our sample contain additional gas giant companions inside $1 \mathrm{au}$ that were not included in our estimate of the frequency of exterior long-period companions. These four inner gas giants are located immediately adjacent to and, in some cases, between the super-Earths in these systems, providing additional support for the idea that the presence of gas giants does not disrupt super-Earth formation. It is likely that these inner gas giants with super-Earth companions did not undergo large-scale migration: high-eccentricity migration of any kind would destroy the orbits of the inner planets (e.g., Mustill et al. 2015; Antonini et al. 2016), and disk-driven migration would lead to resonant locking among the giant planets and the superEarths, resulting in an orbital architecture reminiscent of the Galilean satellites.

If the scenario for the solar system presented in Batygin \& Laughlin (2015) is correct, this would also suggest that the long-period gas giant companions in these systems did not undergo large-scale inward migration. A gas giant undergoing long-range migration (in Batygin \& Laughlin 2015, Jupiter migrated from 6 to $1.5 \mathrm{au}$ ) can capture solid materials in resonance, driving both the inward transport and the excitation of eccentricities. The ensuing collisional cascade among the resonant planetesimals shuttles newly formed super-Earths inward onto their host stars. In this scenario, the total mass participating in this cascade must be large enough to appreciably alter the orbital semi-major axes of the inner super-Earths (i.e., it must have a total mass comparable to that of the planets). The amount of solids participating in the cascade depends on the distance over which the outer gas giants migrate, $M_{\text {swarm }} \sim f_{\text {solid }} \times$ (nebula mass) $\times$ (migration distance/size of the disk), where we assume a gas disk surface density profile of $\propto 1 / r$, with $r$ being the stellocentric distance, and $f_{\text {solid }}$ is the solid-to-gas mass ratio. If, for example, the outer gas giant migrates only $\sim 1$ au for $f_{\text {solid }}=1 / 100$, a nebula mass of $0.01 M_{\odot}$, and a disk size of $30 \mathrm{au}, M_{\text {swarm }} \sim 1 M_{\oplus}$. This is not enough mass to shepherd inner super-Earths onto a host star, rendering this mechanism ineffective in systems where giant planets remain relatively close $(\sim<1 \mathrm{au})$ to their formation locations.

\subsubsection{A Single Long-period Gas Giant is Unlikely to Destroy Inner Super-Earths}

Most systems we identify with significant evidence for an outer companion (see Tables 3 and 5) have strongly coupled inner planets that are resilient against the perturbation of an outer companion (i.e., their mutual secular precession frequency is higher than the outer perturber's precession frequency). Following the procedure outlined in Pu \& Lai (2018), we find that the rms eccentricities (and mutual inclinations) from the secular perturbation by an outer gas giant are limited to, at maximum, just a few percent over a wide range of perturber eccentricities $(0-0.4)$ and inclinations $\left(0^{\circ}-40^{\circ}\right) .^{7}$ The ability of these inner super-Earth systems to remain stable — and roughly coplanar-against the perturbation of the outer perturber and the robustness of our inferred occurrence rate Jovian companion over a range of eccentricity and inclination distributions (see Section 4.1) suggest that the orbital architecture of inner super-Earths is largely unaffected by the presence of an outer gas giant companion.

One notable exception is Kepler-454, which has an inner super-Earth at $\sim 10$ days and a Jovian companion at $\sim 500$ days, as well as an RV acceleration consistent with an outer giant companion at $\sim 10 \mathrm{au}$. While the innermost super-Earth couples more strongly with its closest Jovian neighbor, this Jovian planet couples more strongly with its outer giant companion. Calculating the expected rms eccentricities from the secular perturbations, we find that an outermost companion with an eccentricity greater than $\sim 0.1$ would drive the inner planets to orbital instability. We expect there to be similar limits on the eccentricities of outer gas giants in systems such as GJ $676 \mathrm{~A}$ and HD 181433, which harbor multiple gas giants. In fact, Campanella et al. (2013) argued that the HD 181433 planets must be in a mean-motion resonance in order to ensure orbital stability. The ability of multiple outer gas giants to dynamically excite inner systems has been studied extensively by Hansen \& Zink (2015) for the specific case of $55 \mathrm{Cnc}$ and more generally by Hansen (2017b). Similar system-by-system stability analyses using full $N$-body calculations would help to place stronger constraints on the eccentricities and inclinations of the possible outer companions we report here.

\subsubsection{Outer Gas Giants Provide Constraints on Protoplanetary Disk Properties}

We next consider whether or not the presence of an outer gas giant can be used to constrain the properties of the protoplanetary disks from which these systems formed. Large solid-mass content is considered one such property, facilitating the growth of grains to planetesimals (e.g., Youdin \& Chiang 2004; Birnstiel et al. 2012), accelerating the growth of cores by pebble accretion (e.g., Ormel \& Kobayashi 2012; Lambrechts \& Johansen 2014; Lin et al. 2018), and speeding up the final assembly by giant impact (e.g., Dawson et al. 2015). ${ }^{8}$ Observationally, both gas giants and super-Earths (here defined as planets with mass of $1-10 M_{\oplus}$ and/or radii of $1-4 R_{\oplus}$ ) are found to occur more frequently around metal-rich stars (Fischer \& Valenti 2005; Petigura et al. 2018). Here we consider whether the metallicity of the host star - used as a proxy of the total solid content in the natal disk-is correlated with the occurrence of gas giant companions to inner super-Earths.

\footnotetext{
7 The strength of the secular perturbation is strongly sensitive to the ratio of the orbital distances of the perturber and the inner planet. In our calculation, we adopt the minimum orbital distance of the perturber and its corresponding mass to quantify the maximum possible forced eccentricities and mutual inclinations. We note that the relatively small separation of the outer companion in the Corot-24 system can drive the system to orbital instability for perturber eccentricities larger than $\sim 0.4$. If we instead sample a range of distances and masses of Corot- $24 \mathrm{~d}$ contained within the $1 \sigma$ probability (see Figure 4 ), the rms eccentricities and inclinations drop to just a few percent.

8 To be more precise, the growth of planetesimals and cores is governed by the "local" concentration of solids; in other words, what matters is the solid-togas mass ratio at the site of such growth, not necessarily the bulk mass ratio.
} 
As a test of this question, we divide our sample into systems that have greater than $3 \sigma$ trends (systems listed in bold in Table 1) and resolved companions (systems listed in Table 3), which we refer to hereafter as "companion systems," and those that do not, referred to as "no-companion systems," and compare the error-weighted averages of the stellar metallicities between these two samples. For the transiting planet sample, we find average metallicities of $0.207 \pm 0.005$ and $0.076 \pm 0.003$ for the companion and no-companion systems, a $22 \sigma$ difference. For the RV sample, the error-weighted average metallicities are $0.346 \pm 0.010$ and $-0.292 \pm 0.005$ for the companion and no-companion systems, a $57 \sigma$ difference. We note that in this comparison, we do not include M stars GJ 3293 and GJ 163. This is because we wanted to use metallicities for M stars derived using only IR techniques, since they are consistent and calibrated on FGK stars, and neither of these M stars had metallicities derived using these techniques. However, at present, the "companion" and "no-companion" RV sample averages are significantly influenced by a small fraction of systems that have small metallicity uncertainties. In addition, a caveat for this comparison for both the RV and the transit samples is the fact that error-weighted averages assume that the scatter in metallicity is simply due to measurement uncertainties as opposed to intrinsic astrophysical scatter, which is not the case here given the typically small metallicity uncertainties. Furthermore, these comparisons involve small sample sizes, which is not explicitly accounted for in these error-weighted averages. We thus compare these distributions using the Anderson-Darling test, which takes both the intrinsic astrophysical dispersion of the metallicities and sample size into account. Before applying this test, we remove from the sample all $\mathrm{M}$ stars, which typically had large uncertainties on stellar metallicities in comparison to other stars in the sample, since this test becomes more appropriate the more uniform the sample and the smaller the measurement uncertainties. After applying the Anderson-Darling test, we find that the probability of the companion/no-companion samples coming from the same parent distribution is $p$-val $=0.0027$ and 0.0286 for the RV and transiting samples, corresponding to a significance of $3.0 \sigma$ and $2.2 \sigma$, respectively. While the significance of this comparison is substantially lower than that estimated from comparing the error-weighted averages, given the potential biases of the error-weighted averages presented above, this is a more appropriate assessment of the significance of the differences between the companion and no-companion metallicities. We conclude that these comparisons for the RV and transit samples suggest that super-Earth systems around metal-rich stars are more likely to have outer companions than their metal-poor counterparts. We plot the distribution of metallicities for companion/no-companion systems for each sample in Figure 7 and the metallicities of both RV and transiting samples versus trend significance in Figure 8. We note that the stars in the RV sample typically have lower masses and correspondingly lower metallicities than stars in the transiting planet sample, with an error-weighted average metallicity of $-0.165 \pm 0.004$ and $0.109 \pm 0.003$ for the RV and transiting samples, respectively.

We next consider whether or not there is any correlation between the presence of an outer gas giant companion and the mass of the host star. Observations of young stars indicate that disk mass appears to be correlated with stellar mass, albeit with large intrinsic variance (Andrews et al. 2013;
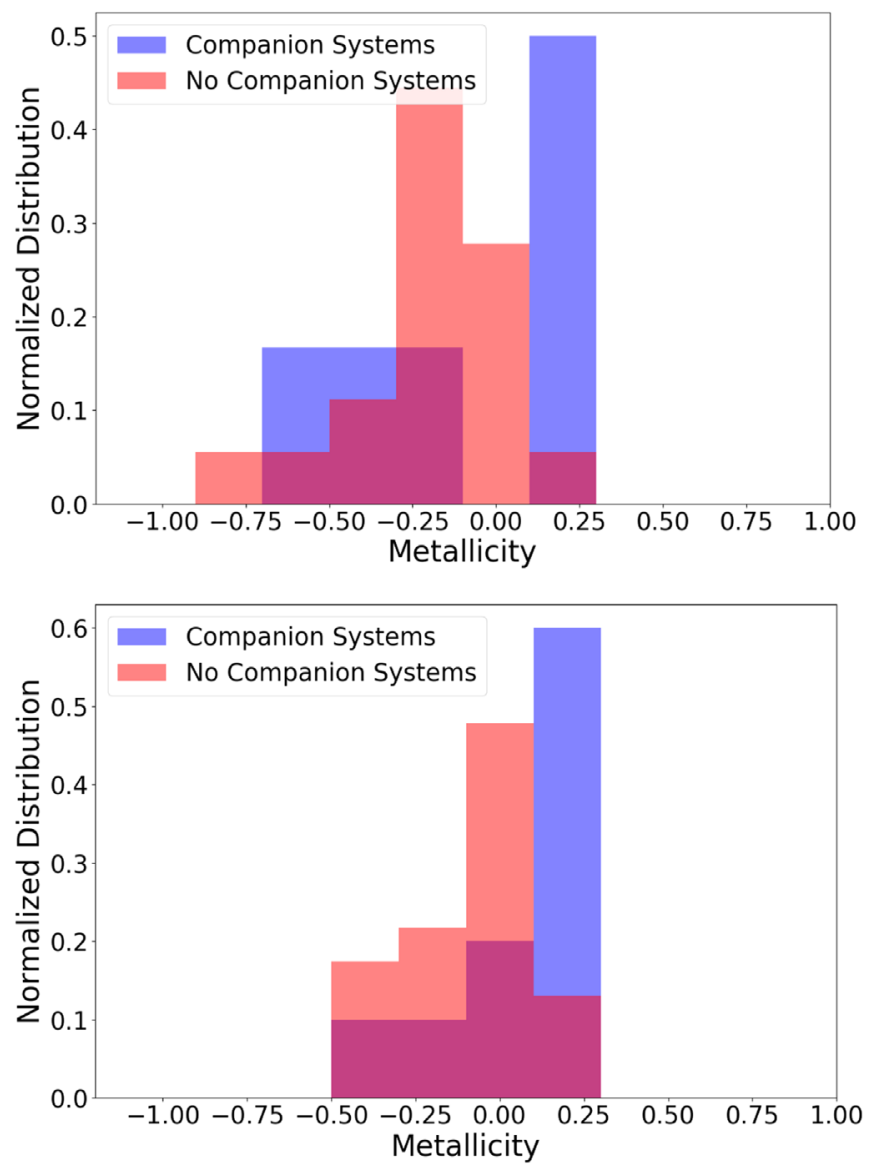

Figure 7. Distributions of stellar metallicities for systems with and without $>3 \sigma$ trends and resolved companions. Top: RV-only sample with 6 systems in the "companion" sample and 19 systems in the "no-companion" sample. Bottom: Transit-only sample with 10 systems in the "companion" sample and 24 systems in the "no-companion" sample.

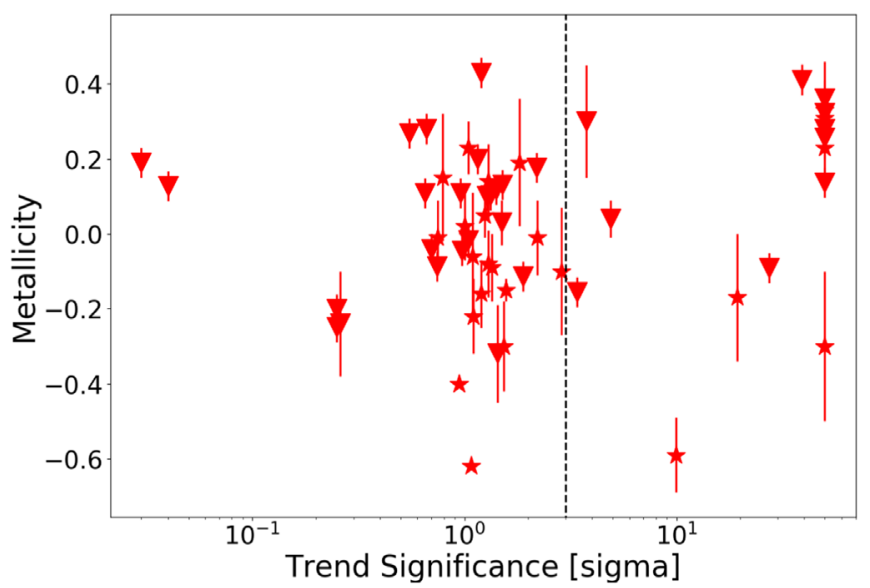

Figure 8. Stellar metallicities plus uncertainties vs. trend significance for the $\mathrm{RV}$ sample (stars) and the transiting sample (triangles). The black dashed line indicates a $3 \sigma$ trend. We assign all resolved companions a significance of $50 \sigma$ in order to include them on this plot.

Pascucci et al. 2016). As with stellar metallicity, we expect that disks with higher overall masses will have a correspondingly higher surface density of solids. However, the benefits of this higher surface density for giant planet formation may be partially negated by the shorter average lifetimes of disks around more massive stars (Ribas et al. 2015). This might 
affect our estimates of the companion frequency in RV versus transiting planet systems, as these two samples have different stellar mass distributions. We find that while only one star in the transiting planet sample is an M dwarf (LHS 1140) out of 34 systems, 10 targets in the RV sample are $M$ dwarfs (out of 25 ). We calculate the occurrence rates for the combined RV and transiting planet sample with and without the $M$ stars only over an integration range of $0.5-20 M_{\text {Jup }}$ and 1-20 au and find occurrence rates of $37 \% \pm 8 \%$ and $44 \% \pm 17 \%$, respectively. These occurrence rates are consistent with the occurrence rate of the total sample of $39 \% \pm 7 \%$ to $<0.1 \sigma$ and $0.3 \sigma$, respectively.

\section{Conclusions}

We collected published RV data for a sample of 65 systems hosting at least one inner super-Earth planet in order to search for massive, long-period companions. We detect these distant companions as long-term trends in the RV data when the orbital period of the companion is shorter than the system RV baseline. Out of our sample of 65 systems, we found 14 systems that had statistically significant trends. Two of these systems had resolved stellar companions that could potentially have caused the observed trends, while three more systems had trends that were likely due to stellar activity. We removed these five systems from subsequent analysis, leaving nine systems with statistically significant trends indicating the presence of an outer companion. Three of these trends are identified here for the first time, while six were previously reported in the literature. We also identify 10 previously published resolved gas giant companions ( $>0.5 M_{\text {Jup }}$ and 1-20 au) in our sample of systems. We report two new candidate planets in systems HD 156668 and HD 175607 but do not include these in our statistical study, as they lie below our mass cutoff with minimum masses of 31 and $24 M_{\oplus}$, respectively. We also recover a fully resolved periodic signal in HD 1461 that appears to be caused by stellar activity, as reported in Bryan et al. (2016).

We compute two-dimensional probability distributions in mass and semi-major axis space for each system in our sample with an RV trend, where we use the duration and shape of the trends to place lower limits on allowed ranges of mass and separation. We use a combination of new and archival AO imaging at infrared wavelengths to place a corresponding upper limit on the allowed masses and separations of these companions. We find that stellar metallicities of systems with gas giant companions are significantly higher than those without companions for both the transiting and RV planet samples, in good agreement with the well-established metallicity correlation from RV surveys of field stars.

We fit the observed companion distributions with a double power law in mass and semi-major axis and integrate this power law over $0.5-20 M_{\text {Jup }}$ and 1-20 au to find an occurrence rate of $39 \% \pm 7 \%$. We then compare our occurrence rate for these companions to similar occurrence rates for long-period gas giant planets from RV surveys of Sun-like field stars. We find that super-Earth systems appear to have more gas giant companions than we would expect to see by chance alone, even after accounting for the additional uncertainty introduced by our inability to pinpoint the precise locations of these companions for systems with RV trends. The high occurrence rate of long-period ( $>1 \mathrm{au}$ ) gas giants in super-Earth systems in turn implies that a significant majority of the long-period gas giants identified in RV surveys of field stars likely host inner super-Earths. We therefore conclude that the presence of an outer gas giant does not hinder super-Earth formation, as proposed in some previous theoretical studies. To the contrary, our data suggest that these companions may either actively facilitate super-Earth formation or simply serve as a fossil record of early disk conditions that were particularly favorable for planet formation over a wide range of semi-major axes.

This work was supported by NSF CAREER grant 1555095 and based in part on observations made at the W. M. Keck Observatory. We extend special thanks to those of Hawaiian ancestry on whose sacred mountain of Maunakea we are privileged to be guests. E.J.L. is supported by the Sherman Fairchild Fellowship from Caltech.

\section{ORCID iDs}

Marta L. Bryan (iD https://orcid.org/0000-0002-6076-5967

Eve J. Lee (iD https://orcid.org/0000-0002-1228-9820

B. J. Fulton (i) https://orcid.org/0000-0003-3504-5316

Henry Ngo (1D https://orcid.org/0000-0001-5172-4859

Tiffany Meshkat (iD https://orcid.org/0000-0001-6126-2467

\section{References}

Affer, L., Micela, G., Damasso, M., et al. 2016, A\&A, 593, A117 Almenara, J. M., Astudillo-Defru, N., Bonfils, X., et al. 2015, A\&A, 581, L7 Alonso, R., Moutou, C., Endl, M., et al. 2014, A\&A, 567, A112 Andrews, S. M., Rosenfeld, K. A., Kraus, A. L., \& Wilner, D. J. 2013, ApJ, 771,129

Anglada-Escudé, G., Amado, P. J., Barnes, J., et al. 2016, Natur, 536, 437 Anglada-Escudé, G., Arriagada, P., Vogt, S. S., et al. 2012, ApJL, 751, L16 Anglada-Escudé, G., \& Tuomi, M. 2012, A\&A, 548, A58

Anglada-Escudé, G., Tuomi, M., Gerlach, E., et al. 2013, A\&A, 556, A126 Antonini, F., Hamers, A. S., \& Lithwick, Y. 2016, AJ, 152, 174

Astudillo-Defru, N., Bonfils, X., Delfosse, X., et al. 2015, A\&A, 575, A119 Astudillo-Defru, N., Forveille, T., Bonfils, X., et al. 2017, A\&A, 602, A88

Baraffe, I., Chabrier, G., Allard, F., \& Hauschildt, P. H. 1998, A\&A, 337, 403 Batygin, K., \& Laughlin, G. 2015, PNAS, 112, 4214

Becker, J. C., \& Adams, F. C. 2017, MNRAS, 468, 549

Berta-Thompson, Z. K., Irwin, J., Charbonneau, D., et al. 2015, Natur, 527, 204

Birnstiel, T., Klahr, H., \& Ercolano, B. 2012, A\&A, 539, A148

Bonfils, X., Delfosse, X., Udry, S., et al. 2005, A\&A, 442, 635

Bonfils, X., Gillon, M., Forveille, T., et al. 2011, A\&A, 528, A111

Bonfils, X., Lo Curto, G., Correia, A. C. M., et al. 2013, A\&A, 556, A110

Borucki, W. J., Agol, E., Fressin, F., et al. 2013, Sci, 340, 587

Borucki, W. J., Koch, D. G., Batalha, N., et al. 2012, ApJ, 745, 120

Bouchy, F., Mayor, M., Lovis, C., et al. 2009, A\&A, 496, 527

Ribas, Á., Bouy, H., \& Merín, B. 2015, A\&A, 576, A52

Bowler, B. P. 2016, PASP, 128, 102001

Bowler, B. P., Johnson, J. A., Marcy, G. W., et al. 2010, ApJ, 709, 396

Bowler, B. P., \& Nielsen, E. L. 2018, in Handbook of Exoplanets, ed. H. Deeg \& J. Belmonte (Cham: Springer), 155

Brewer, J. M., \& Fischer, D. A. 2018, ApJS, 237, 38

Brewer, J. M., Fischer, D. A., Valenti, J. A., \& Piskunov, N. 2016, ApJS, 225, 32

Bryan, M. L., Knutson, H. A., Howard, A. W., et al. 2016, ApJ, 821, 89

Buchhave, L. A., Bizzarro, M., Latham, D. W., et al. 2014, Natur, 509, 593

Buchhave, L. A., Dressing, C. D., Dumusque, X., et al. 2016, AJ, 152, 160

Butler, R. P., Vogt, S. S., Laughlin, G., et al. 2017, AJ, 153, 208

Campanella, G., Nelson, R. P., \& Agnor, C. B. 2013, MNRAS, 433, 3190

Cayrel de Strobel, G., Soubiran, C., \& Ralite, N. 2001, A\&A, 373, 159

Christiansen, J. L., Vanderburg, A., Burt, J., et al. 2017, AJ, 154, 122

Clanton, C., \& Gaudi, B. S. 2016, ApJ, 819, 125

Correia, A. C. M., Couetdic, J., Laskar, J., et al. 2010, A\&A, 511, A21

Crida, A., Morbidelli, A., \& Masset, F. 2006, Icar, 181, 587

Cumming, A., Butler, R. P., Marcy, G. W., et al. 2008, PASP, 120, 531

Dai, F., Winn, J. N., Arriagada, P., et al. 2015, ApJL, 813, L9

Dawson, R. I., Chiang, E., \& Lee, E. J. 2015, MNRAS, 453, 1471 
Delfosse, X., Bonfils, X., Forveille, T., et al. 2013, A\&A, 553, A8 Desch, S. J., Kalyaan, A., \& Alexander, C. M. O. 2018, ApJS, 238, 11 Diaz, R. F., Segransan, D., Udry, S., et al. 2016, A\&A, 585, A134 Dittmann, J. A., Irwin, J. M., Charbonneau, D., et al. 2017, Natur, 544, 333 Dong, S., \& Zhu, Z. 2013, ApJ, 778, 53

Dressing, C. D., \& Charbonneau, D. 2015, ApJ, 807, 45

Dressing, C. D., Charbonneau, D., Dumusque, X., et al. 2015, ApJ, 800, 135 Dumusque, X., Bonomo, A. S., Haywood, R. D., et al. 2014, ApJ, 789, 154

Endl, M., Robertson, P., Cochran, W. D., et al. 2012, ApJ, 759, 19

Ercolano, B., \& Clarke, C. J. 2010, MNRAS, 402, 2735

Fischer, D. A., Marcy, G. W., \& Spronck, J. F. P. 2014, ApJS, 210, 5

Fischer, D. A., \& Valenti, J. 2005, ApJ, 622, 1102

Foreman-Mackey, D., Morton, T. D., Hogg, D. W., Agol, E., \& Schölkopf, B. 2016, AJ, 152, 206

Forveille, T., Bonfils, X., Delfosse, X., et al. 2009, A\&A, 493, 645

Fressin, F., Torres, G., Charbonneau, D., et al. 2013, ApJ, 766, 81

Fulton, B. J., Petigura, E. A., Blunt, S., \& Sinukoff, E. 2018, PASP, 130, 044504

Fulton, B. J., Petigura, E. A., Howard, A. W., et al. 2017, AJ, 154, 109

Fulton, B. J., Weiss, L. M., Sinukoff, E., et al. 2015, ApJ, 805, 175

Furlan, E., Ciardi, D. R., Everett, M. E., et al. 2017, AJ, 153, 71

Gaia Collaboration, Brown, A. G. A., Vallenari, A. , et al. 2018, A\&A, 616, A1

Gaia Collaboration, Brown, A. G. A., Vallenari, A., et al. 2016a, A\&A, 595, A2

Gaia Collaboration, Prusti, T., de Bruijne, J. H. J., et al. 2016b, A\&A, 595, A1 Gautier, T. N., III, Charbonneau, D., Rowe, J. F., et al. 2012, ApJ, 749, 15

Gettel, S., Charbonneau, D., Dressing, C. D., et al. 2016, ApJ, 816, 95

Gillon, M., Demory, B.-O., Van Grootel, V., et al. 2017, NatAs, 1, 0056

Hansen, B. M. S. 2017a, MNRAS, 467, 1531

Hansen, B. M. S. 2017b, MNRAS, 467, 1531

Hansen, B. M. S., \& Zink, J. 2015, MNRAS, 450, 4505

Hasegawa, Y., \& Pudritz, R. E. 2011, MNRAS, 417, 1236

Howard, A. W., Johnson, J. A., Marcy, G. W., et al. 2011, ApJ, 726, 73

Howard, A. W., Marcy, G. W., Fischer, D. A., et al. 2014, ApJ, 794, 51

Howard, A. W., Marcy, G. W., Johnson, J. A., et al. 2010, Sci, 330, 653

Howell, S. B., Rowe, J. F., Bryson, S. T., et al. 2012, ApJ, 746, 123

Huang, C. X., Petrovich, C., \& Deibert, E. 2017, AJ, 153, 210

Isaacson, H., \& Fischer, D. 2010, ApJ, 725, 875

Izidoro, A., Raymond, S. N., Morbidelli, A., Hersant, F., \& Pierens, A. 2015, ApJL, 800, L22

Johnson, J. A., Aller, K. M., Howard, A. W., \& Crepp, J. R. 2010, PASP, 122,905

Kass, R. E., \& Raftery, A. E. 1995, J. Am. Stat. Assoc., 90, 773

Kipping, D. M. 2013, MNRAS, 434, L51

Kley, W., \& Nelson, R. P. 2012, ARA\&A, 50, 211

Knutson, H. A., Fulton, B. J., Montet, B. T., et al. 2014, ApJ, 785, 126

Kraus, A. L., Ireland, M. J., Huber, D., Mann, A. W., \& Dupuy, T. J. 2016, AJ, 152,8

Lambrechts, M., \& Johansen, A. 2014, A\&A, 572, A107

Lambrechts, M., Johansen, A., \& Morbidelli, A. 2014, A\&A, 572, A35

Lee, E. J., \& Chiang, E. 2015, ApJ, 811, 41

Lee, E. J., \& Chiang, E. 2016, ApJ, 817, 90

Léger, A., Rouan, D., Schneider, J., et al. 2009, A\&A, 506, 287

Lenzen, R., Hartung, M., Brandner, W., et al. 2003, Proc. SPIE, 4841, 944

Lin, D. N. C., \& Papaloizou, J. 1986, ApJ, 309, 846

Lin, J. W., Lee, E. J., \& Chiang, E. 2018, MNRAS, 480, 4338

Liu, M. C., Fischer, D. A., Graham, J. R., et al. 2002, ApJ, 571, 519

Lo Curto, G., Mayor, M., Benz, W., et al. 2010, A\&A, 512, A48

Lopez, E. D., \& Fortney, J. J. 2014, ApJ, 792, 1

López-Morales, M., Haywood, R. D., Coughlin, J. L., et al. 2016, AJ, 152, 204

Marcy, G. W., Isaacson, H., Howard, A. W., et al. 2014, ApJS, 210, 20

Masset, F. S., Morbidelli, A., Crida, A., \& Ferreira, J. 2006, ApJ, 642, 478

Mayor, M., Bonfils, X., Forveille, T., et al. 2009, A\&A, 507, 487

Mayor, M., Marmier, M., Lovis, C., et al. 2011, arXiv:1109.2497

Meshkat, T., Kenworthy, M. A., Quanz, S. P., \& Amara, A. 2014, ApJ, 780, 17

Montet, B. T., Crepp, J. R., Johnson, J. A., Howard, A. W., \& Marcy, G. W. 2014, ApJ, 781, 28

Morbidelli, A., Bitsch, B., Crida, A., et al. 2016, Icar, 267, 368
Morbidelli, A., \& Crida, A. 2007, Icar, 191, 158

Morbidelli, A., Lunine, J. I., O’Brien, D. P., Raymond, S. N., \& Walsh, K. J. 2012, AREPS, 40, 251

Morbidelli, A., \& Nesvorny, D. 2012, A\&A, 546, A18

Moriarty, J., \& Fischer, D. 2015, ApJ, 809, 94

Mortier, A., Faria, J. P., Santos, N. C., et al. 2016, A\&A, 585, A135

Mustill, A. J., Davies, M. B., \& Johansen, A. 2015, ApJ, 808, 14

Nelson, B. E., Ford, E. B., Wright, J. T., et al. 2014, MNRAS, 441, 442

Neveu-VanMalle, M., Queloz, D., Anderson, D. R., et al. 2016, A\&A, 586, A93

Ngo, H., Knutson, H. A., Bryan, M. L., et al. 2017, AJ, 153, 242

Ngo, H., Knutson, H. A., Hinkley, S., et al. 2015, ApJ, 800, 138

O’Brien, D. P., Morbidelli, A., \& Levison, H. F. 2006, Icar, 184, 39

Ormel, C. W., \& Kobayashi, H. 2012, ApJ, 747, 115

Owen, J. E., \& Wu, Y. 2013, ApJ, 775, 105

Owen, J. E., \& Wu, Y. 2017, ApJ, 847, 29

Pascucci, I., Testi, L., Herczeg, G. J., et al. 2016, ApJ, 831, 125

Pepe, F., Lovis, C., Ségransan, D., et al. 2011, A\&A, 534, A58

Petigura, E. A., Howard, A. W., \& Marcy, G. W. 2013, PNAS, 110, 19273

Petigura, E. A., Howard, A. W., Marcy, G. W., et al. 2017a, AJ, 154, 107

Petigura, E. A., Marcy, G. W., Winn, J. N., et al. 2018, AJ, 155, 89

Petigura, E. A., Sinukoff, E., Lopez, E. D., et al. 2017b, AJ, 153, 142

Pollack, J. B., Hubickyj, O., Bodenheimer, P., et al. 1996, Icar, 124, 62

Pu, B., \& Lai, D. 2018, MNRAS, 478, 197

Queloz, D., Bouchy, F., Moutou, C., et al. 2009, A\&A, 506, 303

Raymond, S. N. 2006, ApJL, 643, L131

Raymond, S. N., \& Izidoro, A. 2017, Icar, 297, 134

Rice, W. K. M., Armitage, P. J., Wood, K., \& Lodato, G. 2006, MNRAS, 373, 1619

Rodriguez, D. R., Duchêne, G., Tom, H., et al. 2015, MNRAS, 449, 3160

Rojas-Ayala, B., Covey, K. R., Muirhead, P. S., \& Lloyd, J. P. 2012, ApJ, 748, 93

Rousset, G., Lacombe, F., Puget, P., et al. 2003, Proc. SPIE, 4839, 140

Rowan, D., Meschiari, S., Laughlin, G., et al. 2016, ApJ, 817, 104

Sahlmann, J., Lazorenko, P. F., Ségransan, D., et al. 2016, A\&A, 595, A77

Sato, T., Okuzumi, S., \& Ida, S. 2016, A\&A, 589, A15

Schlaufman, K. C., \& Laughlin, G. 2010, A\&A, 519, A105

Service, M., Lu, J. R., Campbell, R., et al. 2016, PASP, 128, 095004

Sinukoff, E., Howard, A. W., Petigura, E. A., et al. 2017, AJ, 153, 70

Stassun, K. G., Collins, K. A., \& Gaudi, B. S. 2017, AJ, 153, 136

Suárez Mascareño, A., González Hernández, J. I., Rebolo, R., et al. 2017, A\&A, 597, A108

Tabachnik, S., \& Tremaine, S. 2002, MNRAS, 335, 151

Tanner, A. M., Gelino, C. R., \& Law, N. M. 2010, PASP, 122, 1195

Torres, G. 1999, PASP, 111, 169

Tsiganis, K., Gomes, R., Morbidelli, A., \& Levison, H. F. 2005, Natur, 435,459

Uehara, S., Kawahara, H., Masuda, K., Yamada, S., \& Aizawa, M. 2016, ApJ, 822,2

Vogt, S. S., Butler, R. P., Marcy, G. W., et al. 2005, ApJ, 632, 638

Vogt, S. S., Wittenmyer, R. A., Butler, R. P., et al. 2010, ApJ, 708, 1366

Wallace, J., Tremaine, S., \& Chambers, J. 2017, AJ, 154, 175

Walsh, K. J., Morbidelli, A., Raymond, S. N., O’Brien, D. P., \& Mandell, A. M. 2011, LPI, 1608, 2585

Weiss, L. M., \& Marcy, G. W. 2014, ApJL, 783, L6

Whipple, F. L. 1972, in From Plasma to Planet, ed. A. Elvius (New York: Wiley), 211

Wittenmyer, R. A., Butler, R. P., Tinney, C. G., et al. 2016, ApJ, 819, 28

Wittenmyer, R. A., Tuomi, M., Butler, R. P., et al. 2014, ApJ, 791, 114

Wright, J. T., Marcy, G. W., Butler, R. P., \& Vogt, S. S. 2004, ApJS, 152, 261

Yasui, C., Kobayashi, N., Tokunaga, A. T., Saito, M., \& Tokoku, C. 2010, ApJL, 723, L113

Youdin, A. N., \& Chiang, E. I. 2004, ApJ, 601, 1109

Zhao, L., Fischer, D. A., Brewer, J., Giguere, M., \& Rojas-Ayala, B. 2018, AJ, 155,24

Zhu, W., Petrovich, C., Wu, Y., Dong, S., \& Xie, J. 2018a, ApJ, 860, 101

Zhu, W., Petrovich, C., Wu, Y., Dong, S., \& Xie, J. 2018b, ApJ, 860, 101

Zhu, W., \& Wu, Y. 2018, AJ, 156, 92 